\title{
Tropical montane forests are a larger than expected global carbon store
}

\author{
D. V. Spracklen ${ }^{1}$ and R. Righelato ${ }^{2}$ \\ ${ }^{1}$ School of Earth and Environment, University of Leeds, Leeds, LS2 9JT, UK \\ ${ }^{2}$ Environmental Systems Science Centre, University of Reading, Reading, RG6 6AL, UK \\ Correspondence to: D. V. Spracklen (dominick@env.leeds.ac.uk)
}

Received: 10 September 2013 - Published in Biogeosciences Discuss.: 3 December 2013

Revised: 7 April 2014 - Accepted: 10 April 2014 - Published: 23 May 2014

\begin{abstract}
Tropical montane forests (TMFs) are recognized for the provision of hydrological services and the protection of biodiversity, but their role in carbon storage is not well understood. We synthesized published observations $(n=94)$ of above-ground biomass (AGB) from forest inventory plots in TMFs (defined here as forests between $23.5^{\circ} \mathrm{N}$ and $23.5^{\circ} \mathrm{S}$ with elevations $\geq 1000 \mathrm{~m}$ a.s.l.). We found that mean (median) AGB in TMFs is 271 (254) $t$ per hectare of land surface. We demonstrate that AGB declines moderately with both elevation and slope angle but that TMFs store substantial amounts of biomass, both at high elevations (up to $3500 \mathrm{~m}$ ) and on steep slopes (slope angles of up to $40^{\circ}$ ). We combined remotely sensed data sets of forest cover with high resolution data of elevation to show that $75 \%$ of the global planimetric (horizontal) area of TMF are on steep slopes (slope angles greater than $27^{\circ}$ ). We used our remote sensed data sets to demonstrate that this prevalence of steep slopes results in the global land surface area of TMF (1.22 million $\left.\mathrm{km}^{2}\right)$ being $40 \%$ greater than the planimetric area that is the usual basis for reporting global land surface areas and remotely sensed data. Our study suggests that TMFs are likely to be a greater store of carbon than previously thought, highlighting the need for conservation of the remaining montane forests.
\end{abstract}

\section{Introduction}

Tropical montane forests (TMFs) are important for the provision of ecosystem services particularly water (Martínez et al., 2009) and biodiversity (Martínez et al., 2009; Gentry, 1992), but their role in global carbon storage is less well known (Bruijnzeel and Veneklaas, 1998). In lowland tropical forests there have been recent advances in our understanding of above-ground biomass (AGB) storage, through synthesis of data from forest inventory plots (Baker et al., 2004; Malhi et al., 2006; Gibbs et al., 2007; Saatchi et al., 2007; Slik et al., 2010; de Castilho et al., 2006; Lewis et al., 2013) and application of remote sensing techniques (Asner et al., 2010; Saatchi et al., 2011; Baccini et al., 2012; Simard et al., 2011). However, knowledge of AGB storage in TMFs is still quite poor: persistent cloud cover and steep terrain makes remote sensing difficult (Simard et al., 2011; Castel et al., 2001), there is a paucity of plot-based data which are difficult to acquire on steep slopes (Malhi et al., 2006), and few attempts have been made to synthesize the available observations. Airborne imaging spectroscopy and lidar has recently been applied to quantify changes in forest structure along elevational gradients (Asner et al., 2012, 2014), and is a promising technique to further our understanding of AGB in TMFs. TMF soils can also contain large amounts of carbon, similar in magnitude to the amount of AGB (e.g. Moser et al., 2011; Álvarez-Arteaga et al., 2013; Omoro et al., 2013; Leuschner et al., 2013). Our study focuses on synthesizing AGB from forest inventory plots, since insufficient data are available to include below-ground carbon as a parameter in our analysis.

The higher elevations of TMFs result in changes to many important environmental variables including temperature, rainfall, cloud cover, incoming solar radiation, wind speed, nutrient inputs and soil type (Benner et al., 2010; Bruijnzeel et al., 2010). The impact of these environmental variables on biomass storage is not well known. TMFs are also commonly located on steep slopes, impacting forest structure through altering access to space and light resources (Robert, 2003) and through altering the incidence of landslides (Dislich and 
Huth, 2012). TMFs typically have lower canopy height than lowland forests (Kitayama and Aiba, 2002; Leuschner et al., 2007, 2014; Fisher et al., 2013; Girardin et al., 2014a; Unger et al., 2012; Asner et al., 2014) which may be expected to reduce AGB storage per tree. Some studies along elevational transects in the Andes have reported increasing stem density with elevation, but no trends in basal area (Girardin et al., 2014a). In contrast, Unger et al. (2012) found decreasing stem density and increasing basal area with increasing elevation in the Andes in Ecuador. Leaf area index tends to decline with increasing elevation (Leuschner et al., 2007, 2013; Moser et al., 2007; Unger et al., 2012, 2013) although some studies report little or no trend (Fischer et al., 2013).

Previous studies of AGB along elevational transects have found declining (Kitayama and Aiba, 2002; Raich et al., 2006; Girardin et al., 2010, 2014a; Leuschner et al., 2013), increasing (Rai and Proctor, 1986) or no (Culmsee et al., 2010; Unger et al., 2012; Girardin et al., 2014a) relationship with elevation. Regional studies suggest that elevation may not be the most important variable in explaining the variability in AGB (Slik et al., 2010; Leuschner et al., 2007), with rainfall and soil characteristics explaining more of the variability in AGB across Borneo compared to elevation. Previous analysis has mainly focused on lowland tropical forests and has correlated AGB with temperature (Raich et al., 2006), rainfall (Malhi et al., 2006; Saatchi et al., 2007; Slik et al., 2010), soils (Malhi et al., 2006; Saatchi et al., 2007; Slik et al., 2010), slope angle (Mascaro et al., 2011) and specifics related to the tree community (Baker et al., 2004). Below-ground carbon in TMFs varies widely, with levels of soil carbon reported from as low as $8.5 \mathrm{tha}^{-1}$ (Girardin et al., 2013) to $257 \mathrm{t} \mathrm{ha}^{-1}$ (Delaney et al., 1997). Fine root biomass increases along elevational transects in the Andes (Kitayama and Aiba, 2002; Leuschner et al., 2007; Moser et al., 2011; Girardin et al., 2013).

To improve our understanding of AGB storage in TMFs we synthesized estimates of live AGB from forest inventory plots that have been reported in the peer-reviewed literature. We explored the role of topographical and climatological variables in controlling AGB. We then used satellite remote sensing observations of pan-tropical forest cover and topography to explore the impact of slope angle on AGB storage.

\section{Methods}

\subsection{Forest inventory plots}

We synthesized peer-reviewed studies of AGB storage in TMFs, where AGB had been estimated from studies of forest plots either using established allometric equations or regressions whose development is described by the authors. We defined TMFs as forests between latitudes of $23.5^{\circ} \mathrm{N}$ and $23.5^{\circ} \mathrm{S}$ and at altitudes $\geq 1000 \mathrm{~m}$ a.s.l. We used AGB data from intact tropical forest sites with little or no sign of human disturbance, described variously as "primary" or "old growth". We also included secondary forest sites where the last disturbance was thought to be at least 40 years old. Where available we also synthesized topographical (elevation and slope angle) and climatological variables (annual mean temperature and annual mean rainfall) for the same plots. Our study focuses on AGB, since insufficient data are available to include below-ground carbon as a parameter in our analysis.

\subsection{Remote sensed data}

We used remotely sensed data sets to analyse the area and topography of TMFs. To analyse the global extent of TMF, we used a remotely sensed data set of humid tropical forest cover at a resolution of $18.5 \mathrm{~km}$ for the year 2000 (Hansen et al., 2008). This product uses Landsat 7 ETM+ to calibrate the vegetation continuous field (VCF) product from the MODerate-resolution Imaging Spectroradiometer (MODIS) sensor onboard NASA's Terra satellite (Hansen et al., 2003). The VCF is derived from all seven bands of the MODIS sensor and contains proportional estimates for vegetation cover (woody vegetation, herbaceous vegetation and bare ground).

To explore the topography of TMF we used the Global Multi-resolution Terrain Elevation Data 2010 (GMTED2010) 7.5 arc second ( $\sim 250 \mathrm{~m}$ horizontal resolution) mean elevation product. GMTED2010 is based on data from 11 different sources with the primary source being the Shuttle Radar Topography Mission. To calculate the elevation of tropical forests we averaged the GMTED data to the same spatial resolution of the forest cover product. We calculated the angle of slope across the tropics at the native resolution of GMTED. Slope was calculated for each pixel of the digital elevation model (DEM) from the mean height of all the pixel neighbours. Our calculated slope will be a lower limit, primarily due to variability in elevation at spatial resolutions less than $250 \mathrm{~m}$.

\subsection{Topographic adjustment to account for slope}

In the forest plot studies (Sect. 2.1), AGB was typically reported per unit area of the Earth's surface although some studies reported AGB per unit planimetric area (Proctor et al., 1983; de Castilho et al., 2006; Alves et al., 2010; Moser et al., 2011; Mascaro et al., 2011). For the latter studies, we used information on slope angle reported by the study to convert AGB to a surface area basis. Remotely sensed data (Sect. 2.2) report planimetric area.

In both forest plot and remotely sensed studies we converted planimetric area $(P)$ to Earth's surface area $(S)$ using

$S=P / \cos (\theta)$, 
where $\theta$ is the angle of the slope. For the remotely sensed data we calculated slope angle from the spatial variability in elevation (Sect. 2.2).

\section{Results and discussion}

\subsection{Forest plot inventory}

\subsubsection{Methodological issues}

First, we explored the impact of methodological issues (forest plot area and the use of different allometric equations) on the AGB reported by the studies. Table 1 gives details on the TMF plot studies synthesized in this analysis. Across our TMF data set, the average total plot area for each AGB in the data set was 0.68 ha (median $=0.3 \mathrm{ha}$ ). Here, the total forest plot area is calculated as the total area of the forest plots for each study at each elevation. Previous work has suggested that small plot size may result in overestimates of AGB (Clark et al., 2001). Our synthesized TMF plots do not show such a bias: mean AGB in TMFs with total plot area $<0.25$ ha was $243 \mathrm{tha}^{-1}(n=44)$ compared to $295 \mathrm{tha}^{-1}$ $(n=50)$ in TMFs with total plot area $\geq 0.25$ ha, with no significant difference with respect to plot area (Student's $t$ test, $P>0.05)$. In fact, there was a small positive relationship between plot size and AGB per unit area within TMFs although the correlation was not significant $\left(r^{2}=0.04 ; P>0.05\right)$.

Next we explored how the choice of allometric equation might have affected the estimated AGB. Many of the studies we synthesized estimated AGB using established allometric equations based on Chave et al. (2005). The majority of the TMF studies in our data set used allometric equations based on tree diameter and tree height $(n=71)$, with fewer studies estimating AGB based only on tree diameter $(n=20)$. We found that the mean AGB in TMF studies where allometric equations included diameter and height $\left(239 \mathrm{tha}^{-1}\right.$, $n=71$ ) was significantly less (Student's $t$ test, $P<0.01$ ) than in studies where the allometric equation was based only on tree diameter and did not include tree height $\left(373 \mathrm{tha}^{-1}\right.$, $n=20$ ). We repeated this analysis at the regional scale. We found that mean AGB in the neotropics was not significantly different $(P>0.05)$ for studies that included both tree diameter and height ( $\left.232 \mathrm{tha}^{-1}, n=40\right)$, compared to studies that only included diameter $\left(266 \mathrm{tha}^{-1}, n=13\right)$. This was in contrast to studies in Asia, where mean AGB was significantly less $(P<0.01)$ in studies that included diameter and height ( $\left.227 \mathrm{tha}^{-1}, n=27\right)$ compared to studies that only included diameter $\left(460 \mathrm{tha}^{-1}, n=4\right)$. However, we note that there were few studies in Asia that only included diameter.

Our analysis suggests that allometric equations that are not specifically developed for TMFs and only include tree diameter, could overestimate AGB. In the following analysis we used data from all the TMF studies. Where necessary, we demonstrate that this selection does not affect our overall conclusions.

\subsubsection{Above-ground biomass in TMF}

Figure 1 shows the location of forest plot studies synthesized in this analysis. We synthesized AGB data $(n=94)$ from forest plots across the neotropics (North, Central and South America, $n=56)$, Africa $(n=7)$ and Asia $(n=31)$. Elevation varied from $1000 \mathrm{~m}$ (based on our definition for TMFs) up to $3600 \mathrm{~m}$, with a mean elevation of $1825 \mathrm{~m}$. In our data set, the AGB in TMFs varied from $77 \mathrm{tha}^{-1}$ to $785 \mathrm{tha}^{-1}$ of the Earth's surface with mean (median) storage of 271 (254) tha ${ }^{-1}$. We found no significant difference (Student's $t$ test, $P>0.1$ ) between mean AGB in Asian TMFs $\left(257 \mathrm{tha}^{-1}, n=31\right)$ compared to Neotropical TMFs ( $247 \mathrm{tha}^{-1}, n=56$ ). This is in contrast to regional patterns observed in lowland forests, where Neotropical forests have significantly less AGB than Asian lowland tropical forests (Slik et al., 2010; Paoli et al., 2008), possibly due to the greater proportion of large trees in Asian forests (Slik et al., 2013). Very few data were available for African TMFs, where mean AGB was $527 \mathrm{tha}^{-1}(n=7)$.

To explore the link between elevation and AGB we compared the TMF data synthesized here with AGB data for lowland forests (defined here as forests below $1000 \mathrm{~m}$ elevation). For data on lowland forests we used data from forest plots below $1000 \mathrm{~m}$ elevation across the studies we synthesized in Table 1 and combined this with data from lowland forests that had been synthesized in previous analyses (Malhi et al., 2006; Slik et al., 2010; de Castilho et al., 2006). Figure 2a compares AGB in Asian and Neotropical TMF against that in lowland forests. We found AGB in TMFs to be significantly lower than that in lowland forests both in the neotropics (Student's $t$ test, $P<0.01)$ and Asia $(P<0.01)$ (Fig. 2a).

Across the combined lowland and TMF data $(n=323)$, elevation has a modest control on AGB $\left(r^{2}=0.1, P<0.01\right)$ with a reduction of $\sim 50 \mathrm{t}$ biomass $\mathrm{ha}^{-1}$ for a $1000 \mathrm{~m}$ increase in elevation $\left(\mathrm{AGB}\left(\mathrm{tha}^{-1}\right)=367-0.048 \times\right.$ elevation (m)). Similar relationships were found when the analysis was restricted to the neotropics $(n=197)\left(r^{2}=0.13, P<0.01\right.$, AGB $\left(\mathrm{t} \mathrm{ha}^{-1}\right)=307-0.032 \times$ elevation (m)) (Fig. 2b).

We found a weaker relationship between AGB and elevation when we restricted our analysis to TMFs $(n=$ $\left.94, r^{2}=0.04, P>0.05\right)$. Indeed, within TMFs we found no significant difference (Student's $t$ test, $P>0.05$ ) between carbon storage in upper-montane (elevation $\geq 2000 \mathrm{~m}$, $n=33$, mean $=233 \mathrm{tha}^{-1}$ ) compared to lower-montane $\left(1000 \mathrm{~m} \leq\right.$ elevation $<2000 \mathrm{~m}, n=61$, mean $\left.=292 \mathrm{tha}^{-1}\right)$ forests. So whilst TMFs have a lower AGB per unit land surface area compared to lowland forests, montane forests can still store substantial amounts of biomass up to elevations of $3500 \mathrm{~m}$.

Our mean AGB in lower-montane forests agrees well with that reported by Asner et al. (2012), who used 
Table 1. Synthesis from the literature of above-ground biomass (AGB) measurements in tropical montane forests (elevation $\geq 1000 \mathrm{~m}$ a.s.1.). All AGB values are reported on a land surface basis. Where the studies synthesized here include data on lowland forests (elevation $<1000$ ma.s.l.), we include these data in the table below (with the exception of Slik et al. (2010) where we only include data for forests $\geq 1000 \mathrm{~m}$ a.s.1.).

\begin{tabular}{|c|c|c|c|c|c|c|c|c|c|}
\hline Location & Lat., Long. & $\begin{array}{l}\text { Elevation } \\
(\mathrm{m})\end{array}$ & $\begin{array}{l}\text { AGB } \\
\left(\text { tha }^{-1}\right)\end{array}$ & $\begin{array}{l}\text { MAT } \\
\left({ }^{\circ} \mathrm{C}\right)\end{array}$ & $\begin{array}{l}\text { Rainfall } \\
\left(\mathrm{mm} \mathrm{yr}^{-1}\right)\end{array}$ & $\begin{array}{l}\text { Slope } \\
\left({ }^{\circ}\right)\end{array}$ & $\begin{array}{l}\text { Plot size } \\
(\mathrm{Ha})\end{array}$ & Equation & Reference \\
\hline $\begin{array}{l}\text { Lowland forest, } \\
\text { Puerto Rico }\end{array}$ & $\begin{array}{l}\sim 18.4^{\circ} \mathrm{N} \\
\sim 66.1^{\circ} \mathrm{W}\end{array}$ & 320 & 226 & - & - & - & 0.1 & Scatena et al. (1993)*14 & Scatena et al. (1993) \\
\hline $\begin{array}{l}\text { Montane moist forests, } \\
\text { Andes, Venezuela }\end{array}$ & $\begin{array}{l}8.62^{\circ} \mathrm{N} \\
71.35^{\circ} \mathrm{W}\end{array}$ & 2250 & 409 & 13.5 & 1500 & - & - & - & $\begin{array}{l}\text { Grimm and Fassbender } \\
\text { (1981) }\end{array}$ \\
\hline $\begin{array}{l}\text { Lowland moist forest, } \\
\text { Caimital, Venezuela }\end{array}$ & $9.5^{\circ} \mathrm{N}, 70^{\circ} \mathrm{W}$ & 150 & 308 & 26 & 1500 & & 0.75 & Brown et al. (1989)*15 & Delaney et al. (1997) \\
\hline $\begin{array}{l}\text { Lowland moist forest, } \\
\text { Ticoporo, Venezuela }\end{array}$ & $9^{\circ} \mathrm{N}, 64^{\circ} \mathrm{W}$ & 240 & 396 & 25.5 & 2850 & & 0.75 & Brown et al. (1989)*15 & Delaney et al. (1997) \\
\hline $\begin{array}{l}\text { Montane moist forest, } \\
\text { Rio Grande, Venezuela }\end{array}$ & $9.5^{\circ} \mathrm{N}, 71^{\circ} \mathrm{W}$ & 2400 & 395 & 15 & 2433 & & 0.75 & Brown et al. (1989)*15 & Delaney et al. (1997) \\
\hline $\begin{array}{l}\text { Upper montane wet forest, } \\
\text { Mucuy, Venezuela }\end{array}$ & $10.5^{\circ} \mathrm{N}, 71^{\circ} \mathrm{W}$ & 2820 & 354 & 10.5 & 1968 & & 0.75 & Brown et al. (1989)*16 & Delaney et al. (1997) \\
\hline $\begin{array}{l}\text { Montane wet forest, } \\
\text { Bismarck Range, } \\
\text { Papua New Guinea }\end{array}$ & $6.0^{\circ} \mathrm{S}, 145.18^{\circ} \mathrm{E}$ & 2500 & 310 & 13 & 3980 & - & 0.24 & $\begin{array}{l}\text { Edwards and Grubb } \\
(1977)^{* 10}\end{array}$ & $\begin{array}{l}\text { Edwards and Grubb } \\
\text { (1977) }\end{array}$ \\
\hline $\begin{array}{l}\text { Upper montane wet forest, } \\
\text { South-Ecuador }\end{array}$ & $\sim 4^{\circ} \mathrm{S}, \sim 79^{\circ} \mathrm{W}$ & 2800 & 149 & - & - & - & 0.3 & Feshe et al. (2002) & $\begin{array}{l}\text { Hofstede and Aguiree } \\
\text { (1999) }\end{array}$ \\
\hline $\begin{array}{l}\text { Montane wet forest, } \\
\text { Pacific Slope, } \\
\text { Ecuador }\end{array}$ & $\sim 3^{\circ} \mathrm{S}, \sim 80^{\circ} \mathrm{W}$ & 2300 & 255 & - & - & - & 0.3 & Feshe et al. (2002) & $\begin{array}{l}\text { Hofstede and Aguiree } \\
\text { (1999) }\end{array}$ \\
\hline $\begin{array}{l}\text { Oyacachi Alnus forest, } \\
\text { Cayambe-Coca } \\
\text { Ecological Reserve, } \\
\text { Napo Province } \\
\text { Ecuador }\end{array}$ & $0.22^{\circ} \mathrm{S}, 78.05^{\circ} \mathrm{W}$ & 3200 & 241 & 10.5 & 2250 & - & 0.3 & Feshe et al. (2002) & Feshe et al. (2002) \\
\hline $\begin{array}{l}\text { Pifo Polyepis forest, } \\
\text { Pichincha province, } \\
\text { Ecuador }\end{array}$ & $0.23^{\circ} \mathrm{S}, 78.25^{\circ} \mathrm{W}$ & 3600 & 366 & 8 & 1500 & - & 0.3 & Feshe et al. (2002) & Feshe et al. (2002) \\
\hline $\begin{array}{l}\text { Metrosideros stands, } \\
\text { Hawaii }\end{array}$ & $19.75^{\circ} \mathrm{N}, 155.25^{\circ} \mathrm{W}$ & $\begin{array}{l}700 \\
1660\end{array}$ & $\begin{array}{l}123 \\
81\end{array}$ & $\begin{array}{l}19.5 \\
13.0\end{array}$ & $\begin{array}{l}6000 \\
2600\end{array}$ & - & - & Raich et al. (1997) & Raich et al. (1997) \\
\hline $\begin{array}{l}\text { Montane tropical forest, } \\
\text { Lore Lindu National Park, } \\
\text { Sulawesi, Indonesia }\end{array}$ & $1.44^{\circ} \mathrm{S}, 120.18^{\circ} \mathrm{E}$ & $\begin{array}{l}1050 \\
1400 \\
1800 \\
2400\end{array}$ & $\begin{array}{l}308.7 \\
304.6 \\
301.4 \\
322.6\end{array}$ & $\begin{array}{l}21.3 \\
19.5 \\
17.3 \\
14.1\end{array}$ & $\begin{array}{l}1894 \\
1975 \\
1891 \\
2131\end{array}$ & $\begin{array}{l}5 \\
5 \\
5 \\
5\end{array}$ & $\begin{array}{l}1.44 \\
0.72 \\
0.48 \\
0.48\end{array}$ & Chave et al. $(2005)^{1}$ & Culmsee et al. (2010) \\
\hline $\begin{array}{l}\text { Tropical forest, } \\
\text { SE Peru }\end{array}$ & $\begin{array}{l}12.83^{\circ} \mathrm{N}, 69.27^{\circ} \mathrm{W} \\
12.84^{\circ} \mathrm{N}, 69.28^{\circ} \mathrm{W}\end{array}$ & $\begin{array}{l}194 \\
210\end{array}$ & $\begin{array}{l}330 \\
300\end{array}$ & $\begin{array}{l}26.4 \\
26.4\end{array}$ & $\begin{array}{l}2730 \\
2730\end{array}$ & - & $\begin{array}{l}1 \\
1\end{array}$ & Chave et al. $(2005)^{2}$ & Girardin et al. (2010) \\
\hline $\begin{array}{l}\text { Montane tropical forest, } \\
\text { SE Peru }\end{array}$ & $\begin{array}{l}12.95^{\circ} \mathrm{N}, 71.57^{\circ} \mathrm{W} \\
13.05^{\circ} \mathrm{N}, 71.53^{\circ} \mathrm{W} \\
13.07^{\circ} \mathrm{N}, 71.55^{\circ} \mathrm{W} \\
13.07^{\circ} \mathrm{N}, 71.55^{\circ} \mathrm{W} \\
13.11^{\circ} \mathrm{N}, 71.58^{\circ} \mathrm{W} \\
13.11^{\circ} \mathrm{N}, 71.6^{\circ} \mathrm{W} \\
13.18^{\circ} \mathrm{N}, 71.58^{\circ} \mathrm{E}\end{array}$ & $\begin{array}{l}1000 \\
1500 \\
1855 \\
2020 \\
2720 \\
3020 \\
3025\end{array}$ & $\begin{array}{l}159 \\
205.6 \\
111.2 \\
77.2 \\
131.8 \\
94 \\
129.8\end{array}$ & $\begin{array}{l}20.7 \\
18.8 \\
18 \\
17.4 \\
13.5 \\
11.8 \\
12.5\end{array}$ & $\begin{array}{l}3087 \\
2631 \\
2472 \\
1827 \\
2318 \\
1776 \\
1706\end{array}$ & $\begin{array}{l}- \\
- \\
- \\
- \\
- \\
- \\
-\end{array}$ & $\begin{array}{l}1 \\
1 \\
1 \\
1 \\
1 \\
1 \\
1\end{array}$ & Chave et al. $(2005)^{2}$ & Girardin et al. (2010) \\
\hline $\begin{array}{l}\text { Montane cloud forest, } \\
\text { Monteverde, } \\
\text { Costa Rica }\end{array}$ & $10.3^{\circ} \mathrm{N}, 84.8^{\circ} \mathrm{W}$ & 1480 & $\begin{array}{l}152 \\
523.1\end{array}$ & - & - & $\begin{array}{l}- \\
-\end{array}$ & $\begin{array}{l}1 \\
4\end{array}$ & Nadkarni et al. (2004) & Nadkarni et al. (2004) \\
\hline $\begin{array}{l}\text { Montane cloud forest, } \\
\text { Cordilleria Central, } \\
\text { Costa Rica }\end{array}$ & $10.4^{\circ} \mathrm{N}, 84.0^{\circ} \mathrm{W}$ & $\begin{array}{l}100 \\
300 \\
500 \\
750 \\
1000 \\
1250 \\
1500 \\
1750 \\
2000 \\
2300 \\
2600\end{array}$ & $\begin{array}{l}278 \\
325 \\
261 \\
346 \\
261 \\
145 \\
215 \\
268 \\
271 \\
349 \\
362\end{array}$ & $\begin{array}{l}24 \\
23 \\
22 \\
20.5 \\
19 \\
17.5 \\
16 \\
14.5 \\
13 \\
11 \\
10.5\end{array}$ & $\begin{array}{l}4000 \\
6000 \\
7000 \\
8000 \\
7000 \\
6000 \\
5000 \\
4000 \\
3500 \\
3500 \\
3300\end{array}$ & $\begin{array}{l}0 \\
0 \\
0 \\
0 \\
0 \\
0 \\
0 \\
0 \\
0 \\
0 \\
0\end{array}$ & $\begin{array}{l}1 \\
1 \\
1 \\
1 \\
1 \\
1 \\
1 \\
1 \\
1 \\
1 \\
1\end{array}$ & Baker et al. (2004) ${ }^{11}$ & Lieberman et al. (1996) \\
\hline
\end{tabular}


Table 1. Continued.

\begin{tabular}{|c|c|c|c|c|c|c|c|c|c|}
\hline Location & Lat., Long. & $\begin{array}{l}\text { Elevation } \\
(\mathrm{m})\end{array}$ & $\begin{array}{l}\text { AGB } \\
\left(\mathrm{tha}^{-1}\right)\end{array}$ & $\begin{array}{l}\text { MAT } \\
\left({ }^{\circ} \mathrm{C}\right)\end{array}$ & $\begin{array}{l}\text { Rainfall } \\
\left(\mathrm{mm} \mathrm{yr}^{-1}\right)\end{array}$ & $\begin{array}{l}\text { Slope } \\
\left({ }^{\circ}\right)\end{array}$ & $\begin{array}{l}\text { Plot Area } \\
(\mathrm{Ha})\end{array}$ & Equation & Reference \\
\hline $\begin{array}{l}\text { Porce region, } \\
\text { Columbia }\end{array}$ & $6.75^{\circ} \mathrm{N}, 75.1^{\circ} \mathrm{W}$ & 1200 & 262.5 & 21.5 & - & - & 3.3 & Sierra et al. (2007)*12 & Sierra et al. (2007) \\
\hline $\begin{array}{l}\text { Lore Lindu National Park, } \\
\text { Sulawesi, Indonesia }\end{array}$ & $1.5^{\circ} \mathrm{S}, 120.05^{\circ} \mathrm{E}$ & 1050 & 286 & - & - & 0 & 0.96 & Chave et al. $(2005)^{1}$ & Hertel et al. (2009) \\
\hline $\begin{array}{l}\text { Serra do Mar Mountains, } \\
\text { São Paulo State, } \\
\text { Brazil }\end{array}$ & $23.43^{\circ} \mathrm{S}, 45.12^{\circ} \mathrm{W}$ & $\begin{array}{l}400 \\
400 \\
1000\end{array}$ & $\begin{array}{l}196.3 \\
194.4 \\
216.9\end{array}$ & $\begin{array}{l}- \\
- \\
-\end{array}$ & $\begin{array}{l}- \\
- \\
-\end{array}$ & $\begin{array}{l}20 \\
40 \\
40\end{array}$ & $\begin{array}{l}4 \\
4 \\
4\end{array}$ & Chave et al. $(2005)^{3}$ & Alves et al. (2010) ${ }^{P}$ \\
\hline $\begin{array}{l}\text { Puu Kolekole, } \\
\text { Hawaii, USA }\end{array}$ & $21.15^{\circ} \mathrm{N}, 156.8^{\circ} \mathrm{W}$ & 1210 & 274 & 16 & 2500 & - & 0.03 & Clark et al. (2001) & Clark et al. (2001) \\
\hline $\begin{array}{l}\text { Laupahoehoe, } \\
\text { Hawaii, USA }\end{array}$ & $19.95^{\circ} \mathrm{N}, 155.3^{\circ} \mathrm{W}$ & 1170 & 532 & 16 & 2500 & - & 0.06 & Clark et al. (2001) & Clark et al. (2001) \\
\hline $\begin{array}{l}\text { Kokee, } \\
\text { Hawaii, USA }\end{array}$ & $22.05^{\circ} \mathrm{N}, 159.5^{\circ} \mathrm{W}$ & 1134 & 412 & 16 & 2500 & - & 0.03 & Clark et al. (2001) & Clark et al. (2001) \\
\hline $\begin{array}{l}\text { Kohala, } \\
\text { Hawaii, USA }\end{array}$ & $20.05^{\circ} \mathrm{N}, 155.9^{\circ} \mathrm{W}$ & 1122 & 290 & 16 & 2500 & - & 0.03 & Clark et al. (2001) & Clark et al. (2001) \\
\hline Puerto Rico, USA & $18.42^{\circ} \mathrm{N}, 66^{\circ} \mathrm{W}$ & 750 & 223 & 19.7 & 3725 & 10 & 2.5 & Ovington and Olson (1970) & Frangi and Lugo (1985) \\
\hline $\begin{array}{l}\text { Luquillo Mountains, } \\
\text { Puerto Rico }\end{array}$ & $\sim 18.3^{\circ} \mathrm{N}, \sim 65.8^{\circ} \mathrm{W}$ & $\begin{array}{l}725 \\
450 \\
1000\end{array}$ & $\begin{array}{l}138.5 \\
197.9 \\
82.9\end{array}$ & $\begin{array}{l}20 \\
21 \\
19\end{array}$ & $\begin{array}{l}3725 \\
3000 \\
4300\end{array}$ & $\begin{array}{l}- \\
- \\
-\end{array}$ & $\begin{array}{l}0.8 \\
0.09 \\
0.22\end{array}$ & Weaver and Murphy (1990) & Weaver and Murphy (1990) \\
\hline $\begin{array}{l}\text { Blue Mountains, } \\
\text { Jamaica }\end{array}$ & $18^{\circ} \mathrm{N}, 77^{\circ} \mathrm{W}$ & $\begin{array}{l}1615 \\
1590 \\
1600 \\
1590 \\
1570\end{array}$ & $\begin{array}{l}238 \\
230 \\
237 \\
312 \\
413\end{array}$ & $\begin{array}{l}15.8 \\
15.5 \\
15.5 \\
15.5 \\
15.3\end{array}$ & $\begin{array}{l}2230 \\
2230 \\
2230 \\
2230 \\
2230\end{array}$ & $\begin{array}{l}- \\
- \\
- \\
-\end{array}$ & $\begin{array}{l}0.1 \\
0.1 \\
0.08 \\
0.12 \\
0.04\end{array}$ & Tanner $(1980)^{13}$ & Tanner (1980) \\
\hline Manu National Park, Peru & $12.35^{\circ} \mathrm{S}, 71.52^{\circ} \mathrm{W}$ & 3345 & 126.8 & 11 & 2200 & - & 5.3 & Chave et al. $(2005)^{2}$ & Gibbon et al. (2010) \\
\hline $\begin{array}{l}\text { Montane Atlantic forests, } \\
\text { Rio de Janeiro State, Brazil }\end{array}$ & $21.62^{\circ} \mathrm{S}, 42.08^{\circ} \mathrm{W}$ & $\begin{array}{l}900 \\
600\end{array}$ & $\begin{array}{l}148.4 \\
167.9\end{array}$ & - & $\begin{array}{l}1440 \\
1440\end{array}$ & - & $\begin{array}{l}0.15 \\
0.15\end{array}$ & Cunha et al. (2009) & Cunha et al. (2009) \\
\hline $\begin{array}{l}\text { Podocarpus National Park, } \\
\text { Ecuador }\end{array}$ & $\begin{array}{l}4.1^{\circ} \mathrm{S}, 78.96^{\circ} \mathrm{W} \\
4.1^{\circ} \mathrm{S}, 78.96^{\circ} \mathrm{W} \\
4.0^{\circ} \mathrm{S}, 79.0^{\circ} \mathrm{W} \\
4.0^{\circ} \mathrm{S}, 79.0^{\circ} \mathrm{W} \\
4.1^{\circ} \mathrm{S}, 79.2^{\circ} \mathrm{W}\end{array}$ & $\begin{array}{l}1050 \\
1540 \\
1890 \\
2380 \\
3060\end{array}$ & $\begin{array}{l}256.2 \\
165 \\
148.3 \\
88.1 \\
100\end{array}$ & $\begin{array}{l}18.9 \\
16.7 \\
14.9 \\
12.3 \\
8.6\end{array}$ & $\begin{array}{l}2230 \\
2300 \\
1950 \\
5000 \\
4500\end{array}$ & $\begin{array}{l}26 \\
10 \\
31 \\
28 \\
27\end{array}$ & $\begin{array}{l}0.16 \\
0.16 \\
0.16 \\
0.16 \\
0.16\end{array}$ & Chave et al. $(2005)^{2}$ & $\begin{array}{l}\text { Moser et al. (2011) }{ }^{P} \\
\text { Leuschner et al. }(2007)\end{array}$ \\
\hline $\begin{array}{l}\text { Buenaventura Reserve, } \\
\text { Ecuador }\end{array}$ & $\sim 4^{\circ} \mathrm{S}, \sim 79^{\circ} \mathrm{W}$ & 1000 & 183 & - & - & - & 1 & Chave et al. $(2005)^{* 9}$ & Spracklen et al. (2005) \\
\hline Tapichalaca Reserve, Ecuador & $\sim 4.28^{\circ} \mathrm{S}, \sim 79^{\circ} \mathrm{W}$ & 2250 & 195 & - & - & - & 1 & Chave et al. $(2005)^{* 9}$ & Spracklen et al. (2005) \\
\hline Bannadpare, South India & $12.08^{\circ} \mathrm{N}, 75.7^{\circ} \mathrm{E}$ & 200 & 454 & 27 & 5310 & 4 & 1 & Rai and Proctor $(1986)^{* 17}$ & Rai and Proctor (1986) \\
\hline $\begin{array}{l}\text { Agumbe, Karnataka, } \\
\text { southern India }\end{array}$ & $13.52^{\circ} \mathrm{N}, 75.1^{\circ} \mathrm{E}$ & 575 & 420 & 22.2 & 7670 & 0 & 0.44 & Rai and Proctor $(1986)^{* 17}$ & Rai and Proctor (1986) \\
\hline $\begin{array}{l}\text { Kagneri, Karnataka, } \\
\text { southern India }\end{array}$ & $12.82^{\circ} \mathrm{N}, 75.6^{\circ} \mathrm{E}$ & 500 & 460 & 28.6 & 6100 & 10 & 1 & Rai and Proctor $(1986)^{* 17}$ & Rai and Proctor (1986) \\
\hline $\begin{array}{l}\text { South Bhadra, Karnataka, } \\
\text { southern India }\end{array}$ & $13.25^{\circ} \mathrm{N}, 75.25^{\circ} \mathrm{E}$ & 800 & 649 & 21 & 6520 & 0 & 0.5 & Rai and Proctor $(1986)^{* 17}$ & Rai and Proctor (1986) \\
\hline $\begin{array}{l}\text { Jianfengling } \\
\text { National Natural Reserve, } \\
\text { Hainan, China }\end{array}$ & $18.72^{\circ} \mathrm{N}, 108.88^{\circ} \mathrm{E}$ & $\begin{array}{l}893 \\
867\end{array}$ & $\begin{array}{l}422.2 \\
479.7\end{array}$ & $\begin{array}{l}19.8 \\
19.8\end{array}$ & $\begin{array}{l}2449 \\
2449\end{array}$ & - & $\begin{array}{l}1 \\
0.3\end{array}$ & - & Chen et al. (2010) \\
\hline $\begin{array}{l}\text { Mount Kinabalu, Sabah, } \\
\text { Malaysia }\end{array}$ & $6.08^{\circ} \mathrm{N}, 116.55^{\circ} \mathrm{E}$ & $\begin{array}{l}650 \\
1560 \\
2590 \\
3080 \\
700 \\
1860 \\
2700\end{array}$ & $\begin{array}{l}437 \\
294 \\
308 \\
215 \\
548 \\
238 \\
122\end{array}$ & $\begin{array}{l}24.5 \\
18.5 \\
12.5 \\
10 \\
24 \\
17 \\
12\end{array}$ & $\begin{array}{l}2300 \\
2300 \\
2300 \\
2300 \\
2300 \\
2300 \\
2300\end{array}$ & $\begin{array}{l}19 \\
17 \\
20 \\
27 \\
11 \\
24 \\
22\end{array}$ & $\begin{array}{l}1 \\
0.5 \\
0.25 \\
0.2 \\
1 \\
0.2 \\
0.2\end{array}$ & Yamakur et al. $(1986)^{8}$ & Kitayama and Aiba (2002) \\
\hline Gunung Kinabalu, Sabah & $6.04^{\circ} \mathrm{N}, 116.54^{\circ} \mathrm{E}$ & 1958 & 405.0 & - & - & - & 2.4 & Chave et al. $(2005)^{* 5}$ & Slik et al. (2010) \\
\hline Gunung Mulu, Sarawak & $\begin{array}{l}4.02^{\circ} \mathrm{N}, 114.82^{\circ} \mathrm{E} \\
4.04^{\circ} \mathrm{N}, 114.86^{\circ} \mathrm{E} \\
4.15^{\circ} \mathrm{N}, 114.88^{\circ} \mathrm{E} \\
4.14^{\circ} \mathrm{N}, 114.88^{\circ} \mathrm{E}\end{array}$ & $\begin{array}{l}50 \\
225 \\
170 \\
300\end{array}$ & $\begin{array}{l}250 \\
621.6 \\
469.7 \\
338.6\end{array}$ & $\begin{array}{l}25 \\
24 \\
24 \\
23.5\end{array}$ & $\begin{array}{l}5090 \\
5110 \\
5700 \\
5700\end{array}$ & $\begin{array}{l}0 \\
17 \\
2 \\
27\end{array}$ & $\begin{array}{l}1 \\
1 \\
1 \\
1\end{array}$ & Proctor et al. $(1983)^{7}$ & Proctor et al. $(1983)^{P}$ \\
\hline Long Barang, East Kalimantan & $1.87^{\circ} \mathrm{N}, 115.12^{\circ} \mathrm{E}$ & 1026 & 359.1 & - & - & - & 1.26 & Chave et al. $(2005)^{* 5}$ & Slik et al. (2010) \\
\hline Puak Highlands, East Kalimantan & $2.87^{\circ} \mathrm{N}, 115.70^{\circ} \mathrm{E}$ & 1349 & 765.5 & - & - & - & 4.5 & Chave et al. $(2005)^{* 5}$ & Slik et al. (2010) \\
\hline $\begin{array}{l}\text { Challabamba, } \\
\text { Manu National Park, } \\
\text { Peru }\end{array}$ & $13^{\circ} \mathrm{N}, 71.6^{\circ} \mathrm{W}$ & 3100 & $\begin{array}{l}198 \\
(197)\end{array}$ & - & - & 33 & 0.1 & $\begin{array}{l}\text { Román-Cuesta*6 } \\
\text { et al. (2011) }\end{array}$ & $\begin{array}{l}\text { Román-Cuesta } \\
\text { et al. }(2011)^{\mathrm{c}}\end{array}$ \\
\hline
\end{tabular}


Table 1. Continued.

\begin{tabular}{|c|c|c|c|c|c|c|c|c|c|}
\hline Location & Lat., Long. & $\begin{array}{l}\text { Elevation } \\
(\mathrm{m})\end{array}$ & $\begin{array}{l}\text { AGB } \\
\left(\text { tha }^{-1}\right)\end{array}$ & $\begin{array}{l}\text { MAT } \\
\left({ }^{\circ} \mathrm{C}\right)\end{array}$ & $\begin{array}{l}\text { Rainfall } \\
\left(\mathrm{mm} \mathrm{yr}^{-1}\right)\end{array}$ & $\begin{array}{l}\text { Slope } \\
\left({ }^{\circ}\right)\end{array}$ & $\begin{array}{l}\text { Plot size } \\
(\mathrm{Ha})\end{array}$ & Equation & Reference \\
\hline $\begin{array}{l}\text { Laguna-Acjanaco, } \\
\text { Manu National Park, } \\
\text { Peru }\end{array}$ & $13^{\circ} \mathrm{N}, 71.6^{\circ} \mathrm{W}$ & 3400 & $\begin{array}{l}169 \\
(171)\end{array}$ & - & - & 24 & 0.1 & $\begin{array}{l}\text { Román-Cuesta*6 } \\
\text { et al. (2011) }\end{array}$ & $\begin{array}{l}\text { Román-Cuesta } \\
\text { et al. }(2011)^{c}\end{array}$ \\
\hline $\begin{array}{l}\text { Pahititi, Manu } \\
\text { National Park }\end{array}$ & $13^{\circ} \mathrm{N}, 71.6^{\circ} \mathrm{W}$ & 2920 & $\begin{array}{l}120 \\
(125)\end{array}$ & - & - & 37 & 0.1 & $\begin{array}{l}\text { Román-Cuesta*6 } \\
\text { et al. (2011) }\end{array}$ & $\begin{array}{l}\text { Román-Cuesta } \\
\text { et al. }(2011)^{c}\end{array}$ \\
\hline Sondor National Park & $13^{\circ} \mathrm{N}, 71.6^{\circ} \mathrm{W}$ & 2850 & $\begin{array}{l}241 \\
(236)\end{array}$ & - & - & 17 & 0.1 & $\begin{array}{l}\text { Román-Cuesta*6 } \\
\text { et al. (2011) }\end{array}$ & $\begin{array}{l}\text { Román-Cuesta } \\
\text { et al. }(2011)^{c}\end{array}$ \\
\hline Taita Hills, Kenya & $3.37^{\circ} \mathrm{S}, 38.34^{\circ} \mathrm{E}$ & 1826 & 607 & 18.3 & 1591 & 14 & 0.13 & Chave et al. $(2005)^{* 18}$ & Omoro et al. (2013) \\
\hline Taita Hills, Kenya & $3.47^{\circ} \mathrm{S}, 38.34^{\circ} \mathrm{E}$ & 1535 & 785 & 18.3 & 1591 & 14 & 0.13 & Chave et al. $(2005)^{* 18}$ & Omoro et al. (2013) \\
\hline Taita Hills, Kenya & $3.3^{\circ} \mathrm{S}, 38.5^{\circ} \mathrm{E}$ & 1390 & 767 & 18.3 & 1591 & 14 & 0.27 & Chave et al. $(2005)^{* 18}$ & Omoro et al. (2013) \\
\hline $\begin{array}{l}\text { Sierra Norte Oaxaca, } \\
\text { Mexico }\end{array}$ & $17.83^{\circ} \mathrm{N}, 96.17^{\circ} \mathrm{W}$ & $\begin{array}{l}1500 \\
1950 \\
2050 \\
2400 \\
2500\end{array}$ & $\begin{array}{l}377 \\
271 \\
263 \\
254 \\
444\end{array}$ & $\begin{array}{l}16.5 \\
- \\
- \\
- \\
-\end{array}$ & $\begin{array}{l}5800 \\
- \\
- \\
- \\
-\end{array}$ & $\begin{array}{l}- \\
- \\
- \\
- \\
-\end{array}$ & $\begin{array}{l}0.3 \\
0.3 \\
0.3 \\
0.3 \\
0.3\end{array}$ & Acosta-Mireles et al. (2002)* & $\begin{array}{l}\text { Álvarez-Alteaga } \\
\text { et al. (2013) }\end{array}$ \\
\hline Mt. Rinjani, Lombok & $8.4^{\circ} \mathrm{S}, 116.4^{\circ} \mathrm{E}$ & $\begin{array}{l}1000 \\
1000 \\
1000 \\
1200 \\
1200 \\
1200 \\
1400 \\
1400 \\
1400 \\
1600 \\
1600 \\
1600 \\
1800 \\
1800 \\
1800 \\
2000 \\
2000\end{array}$ & $\begin{array}{l}110 \\
160 \\
230 \\
210 \\
240 \\
270 \\
205 \\
245 \\
255 \\
215 \\
140 \\
110 \\
280 \\
210 \\
205 \\
205 \\
130\end{array}$ & $\begin{array}{l}- \\
- \\
- \\
- \\
- \\
- \\
- \\
- \\
- \\
- \\
- \\
- \\
- \\
- \\
- \\
- \\
-\end{array}$ & $\begin{array}{l}- \\
- \\
- \\
- \\
- \\
- \\
- \\
- \\
- \\
- \\
- \\
- \\
- \\
- \\
- \\
- \\
-\end{array}$ & $\begin{array}{l}22 \\
22 \\
22 \\
22 \\
22 \\
22 \\
22 \\
22 \\
22 \\
22 \\
22 \\
22 \\
22 \\
22 \\
22 \\
22 \\
22\end{array}$ & $\begin{array}{l}0.1 \\
0.1 \\
0.1 \\
0.1 \\
0.1 \\
0.1 \\
0.1 \\
0.1 \\
0.1 \\
0.1 \\
0.1 \\
0.1 \\
0.1 \\
0.1 \\
0.1 \\
0.1 \\
0.1\end{array}$ & Chave et al. $(2005)^{4}$ & Dossa et al. (2013) \\
\hline Uganda BUD-17 & $1.72^{\circ} \mathrm{N}, 31.5^{\circ} \mathrm{E}$ & 1062 & 603.9 & 23.1 & 1326 & - & 1.86 & Chave et al. $(2005)^{4}$ & Lewis et al. (2013) \\
\hline Cameroon TNP-08 & $6.31^{\circ} \mathrm{N}, 9.37^{\circ} \mathrm{E}$ & 1217 & 249.2 & 21.3 & 2145 & - & 1 & Chave et al. $(2005)^{4}$ & Lewis et al. (2013) \\
\hline Tanzania VTA-13 & $5.11^{\circ} \mathrm{N}, 38.6^{\circ} \mathrm{E}$ & 995 & 454.5 & 20.1 & 1847 & - & 1 & Chave et al. $(2005)^{4}$ & Lewis et al. (2013) \\
\hline Tanzania VTA-30 & $6^{\circ} \mathrm{S}, 37.72^{\circ} \mathrm{E}$ & 1012 & 283.5 & 20.9 & 1108 & - & 0.4 & Chave et al. $(2005)^{4}$ & Lewis et al. (2013) \\
\hline Uganda MPG01 & $0.21^{\circ} \mathrm{N}, 32.29^{\circ} \mathrm{E}$ & 1219 & 396.2 & 21.3 & 1286 & - & 0.64 & Chave et al. $(2005)^{4}$ & Lewis et al. (2013) \\
\hline NE Ecuador Jatun Sacha & $0.1^{\circ} \mathrm{S}, 78.02^{\circ} \mathrm{W}$ & $\begin{array}{l}500 \\
1000 \\
1500 \\
2000\end{array}$ & $\begin{array}{l}343 \\
371 \\
354 \\
307\end{array}$ & $\begin{array}{l}22.9 \\
20 \\
17.1 \\
14.3\end{array}$ & $\begin{array}{l}2500 \\
2500 \\
2500 \\
2500\end{array}$ & $\begin{array}{l}- \\
- \\
- \\
-\end{array}$ & $\begin{array}{l}0.8 \\
0.8 \\
0.8 \\
0.8\end{array}$ & Chave et al. $(2005)^{2}$ & Unger et al. (2012) \\
\hline
\end{tabular}

${ }^{1} \mathrm{AGB}=\exp \left[-2.557+0.940 \ln \left(\rho D^{2} H\right)\right]$

$2 \mathrm{AGB}=0.0776\left(\rho D^{2} H\right)^{0.94}$
(1)

$3 \mathrm{AGB}=\exp \left[-2.977+\ln \left(\rho D^{2} H\right)\right]$

${ }^{4} \mathrm{AGB}=0.0509\left(\rho D^{2} H\right)$

${ }^{5} \mathrm{AGB}=\rho \cdot \exp \left(-1.239+1.980 \ln D+0.207(\ln D)^{2}-0.0281(\ln D)^{3}\right)$

${ }^{6} \mathrm{AGB}=0.07 D^{2.417}$

$7 \mathrm{AGB}=1.1 \rho 0.5 \mathrm{AH}$

$8 \mathrm{AGB}=S+B+L$, where $S=0.02903\left(D^{2} H\right)^{0.9813} ; B=0.1192 S^{1.059} ; L=0.09146(S+B)^{0.7266}$

${ }^{9} \mathrm{AGB}=\exp \left[-2.289+2.649 \ln (D)\left(0.021(\ln D)^{2}\right)\right]$

${ }^{10} \mathrm{AGB}=9.541+0.508 D^{2}$

${ }_{11} \mathrm{AGB}=0.6(66.92+16.85 A)$

${ }^{12} \mathrm{AGB}=\exp [-2.286+2.471 \ln D]$

$13 \mathrm{AGB}=\exp [-1.52+1.07 \ln A]$

${ }^{14} \mathrm{AGB}=\exp [2.475 \ln D-2.399]$

$15 \mathrm{AGB}=38.4908-11.7883 D+1.1926 D^{2}$

$16 \mathrm{AGB}=13.2597-4.8945 D+0.6713 D^{2}$

$17 \mathrm{AGB}=\exp [-0.435+2.12 \ln D]$

${ }^{18} \mathrm{AGB}=\rho \cdot \exp \left(-1.499+2.148 \ln D+0.207(\ln D)^{2}-0.0281(\ln D)^{3}\right)$

where $D=$ diameter at breast height (in $\mathrm{cm}$ ), $H=$ total tree height (in m), $A=$ basal area, $\rho=$ wood density (in $\mathrm{g} \mathrm{m}^{-3}$ )

*Allometric equation does not include tree height

$P$ AGB originally reported on planimetric area basis

aiomass converted from carbon using a conversion factor of 2 .

b Stand volume converted to biomass assuming a wood specific density of $0.56 \mathrm{~g} \mathrm{~cm}^{-3}$ which is the mean reported by Culmsee et al. (2010).

${ }^{\mathrm{c}}$ Individual site values are not reported. We calculate AGB values for each site through weighting the average value for all sites with basal area $\times$ height for each site. Weighting using the natural logarithm of (basal

area $\times$ height) changes the AGB values only slightly (reported in parentheses). 
(a)

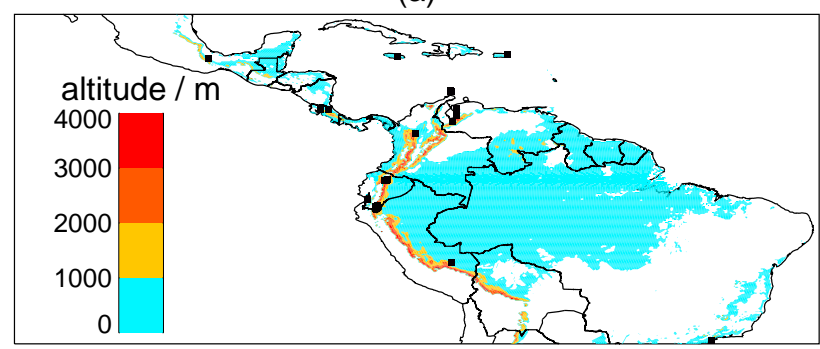

(b)

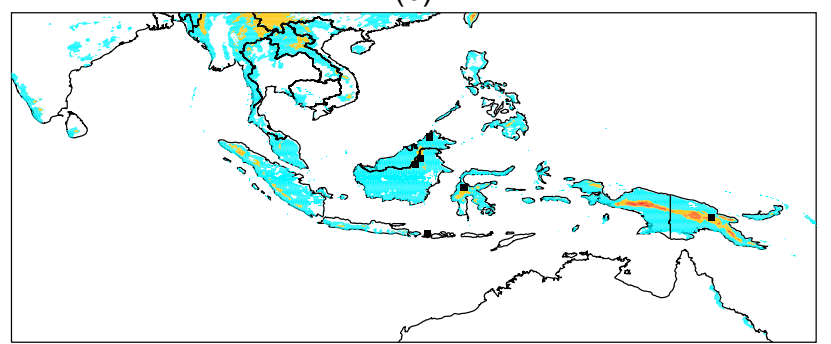

(c)

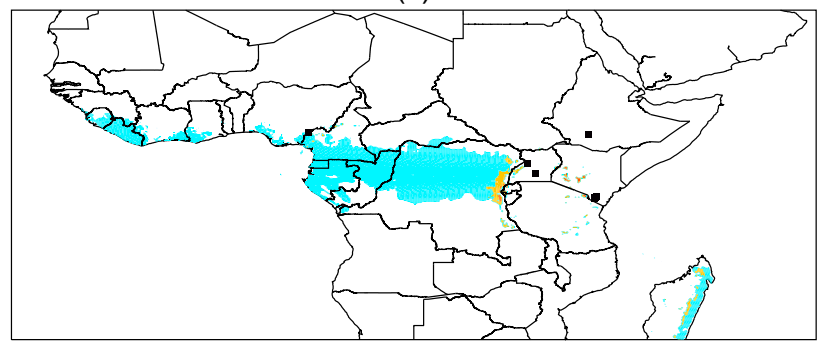

Figure 1. Location of tropical montane forest inventory plots (solid squares) where data of above-ground biomass have been synthesized for this analysis in (a) neotropics (sites in Hawaii are not shown here), (b) Asia and (c) Africa. Note that some squares represent multiple plots. Background colour shows elevation of tropical forests (coloured where vegetation continuous field from MODIS is $>25 \%)$.

an aircraft-borne lidar over Madagascar to estimate AGB of $197 \mathrm{tha}^{-1}$ (assuming a factor 2 conversion between biomass and carbon) in lower-montane forests $(1000 \mathrm{~m} \leq$ elevation $<2000 \mathrm{~m})$. In upper-montane forests $\left(\geq 2000 \mathrm{~m}\right.$ ) our mean AGB was greater than the $82 \mathrm{tha}^{-1}$ reported by Asner et al. (2012) for Madagascar.

In our data set we find substantial variability of AGB across forest plots in both lowland forests $(n=229$, standard deviation $(\sigma)=113 \mathrm{tha}^{-1}$; coefficient of variation $(C V)=0.31)$ and TMFs $\left(\sigma=144 \mathrm{tha}^{-1}, \mathrm{CV}=0.53\right)$. Previous studies have suggested that variability in AGB decreases at higher elevations (Asner et al., 2012, 2014). In our data set, we find that variability in uppermontane forests (elevation $\geq 2000 \mathrm{~m}, n=33, \sigma=106 \mathrm{tha}^{-1}$, $\mathrm{CV}=0.45)$ is less ( $F$ test, $P=0.015)$ than in lowermontane forests $(1000 \mathrm{~m}<$ elevation $<2000 \mathrm{~m}, \quad n=61$, $\left.\sigma=158 \mathrm{tha}^{-1}, \mathrm{CV}=0.54\right)$. However, when we restrict our analysis to studies that include tree height in the allomet- ric equation used to calculate $\mathrm{AGB}$, we find there is no significant difference $(P>0.1)$ in variability between uppermontane forests $\left(n=17, \sigma=106 \mathrm{tha}^{-1}, \mathrm{CV}=0.52\right)$ and lower-montane forests $\left(n=50, \sigma=102 \mathrm{tha}^{-1}, \mathrm{CV}=0.40\right)$. We also find that the variability of AGB in upper montane forests is not significantly different from lowland forests $(F$ test, $P>0.1$ ), with consistent results independent of choice of allometric equation.

We also explored the correlation between AGB and climatological and topographical variables. In our combined data set (TMFs and lowland forests where all variables are available, $n=111$ ) we found modest negative correlation of AGB with slope angle $\left(r^{2}=0.1, P<0.01\right.$, Fig. $\left.2 c\right)$, but no significant correlation $(P>0.05)$ with elevation $\left(r^{2}=0.01\right)$, annual mean mean temperature $\left(r^{2}=0.03\right)$ or rainfall $\left(r^{2}<\right.$ 0.001) (Table 2 and Fig. 3). Therefore we find that the weak correlation between AGB and elevation reported above for all our data, is not significant when we restrict the data set to studies where slope angle, temperature and rainfall are available. We note that elevation is not a direct controlling factor, with many environmental variables varying along elevational gradients (Girardin et al., 2014a). Our analysis is restricted to tropical sites, so elevation and temperature are strongly correlated in our data set $\left(r^{2}=0.96\right)$. When we restricted our analysis to TMFs ( $n=24$, elevations $\geq 1000 \mathrm{~m}$ ), correlations with AGB were slightly stronger: annual mean temperature $\left(r^{2}=0.14, P>0.05\right)$, annual mean rainfall $\left(r^{2}=0.20\right.$, $P=0.03)$, slope angle $\left(r^{2}=0.1, P>0.05\right)$ and elevation $\left(r^{2}=0.07, P>0.05\right)$, but never significant at $P<0.01$ (Table 2).

Lack of strong correlation of AGB with topographic and climatic variables has been previously reported (Slik et al., 2010; Leuschner et al., 2007). Other environmental parameters such as nutrient availability (Fischer et al., 2013; Unger et al., 2012; Homeier et al., 2013), soil properties, ultraviolet light exposure, light exposure, cloud immersion or wind speed may play an important role in AGB storage (Girardin et al., 2014a). Fischer et al. (2013) reported increased nitrogen limitation but decreased phosphorus limitation with increasing elevation in the Peruvian Andes. Studies of net primary productivity along elevational transects display abrupt changes at specific elevations that may be associated with factors such as frequency of cloud immersion controlling light and humidity (Girardin et al., 2010, 2014a). Seasonal variability in net primary productivity at two montane forest sites in the Andes is also linked to solar radiation and cloud immersion (Girardin et al., 2014b) potentially implicating these variables as important drivers of AGB storage. Change in woody species richness, which often declines with increasing elevation, has potential implications for carbon storage (Girardin et al., 2014a). Furthermore, it is possible that soil properties which are known to affect AGB in lowland forests (de Castilho et al., 2006; Paoli et al., 2008) also play a role in TMFs (Unger et al., 2012). We were not able to explore the role of such factors because they were not systematically 

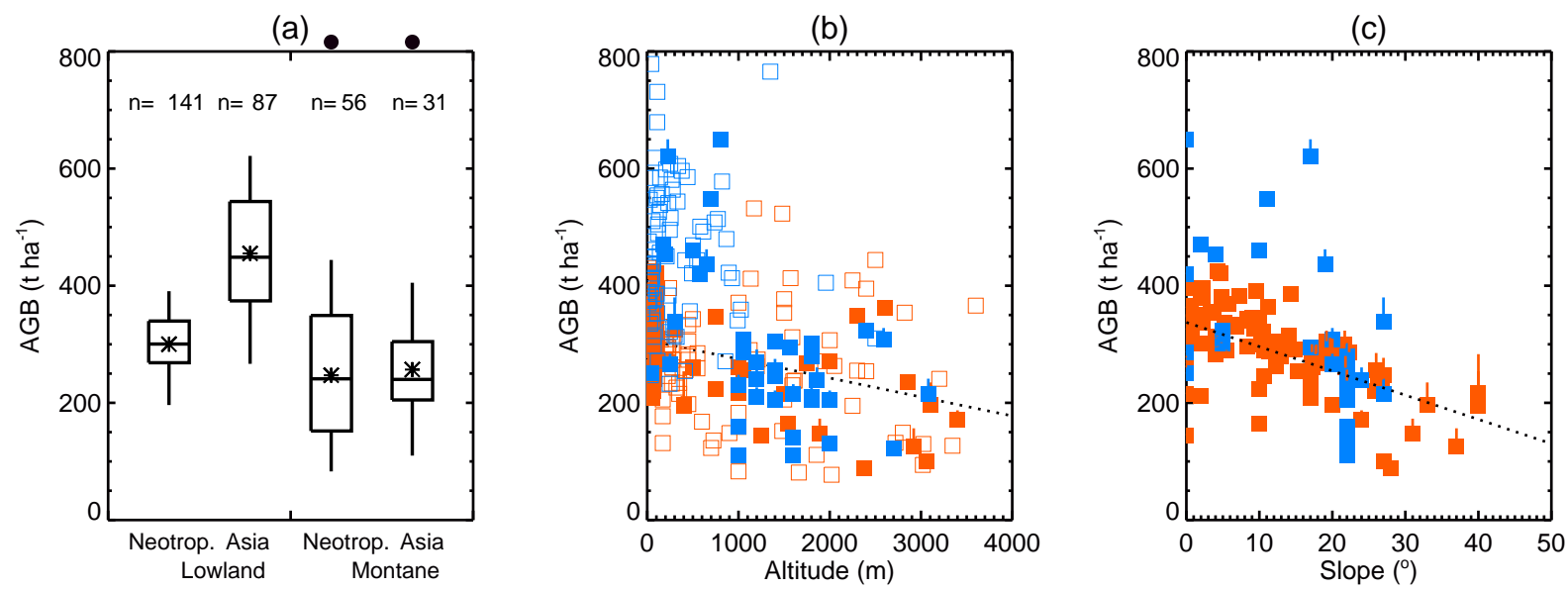

Figure 2. Above-ground biomass (AGB) storage estimated from forest inventory plots. (a) Comparison of AGB (on a land surface area basis) in tropical montane forests (elevation $\geq 1000 \mathrm{~m}$ ) with that in lowland tropical forests (star: mean, line: median, box: 25 th and 75th percentile, whisker: 5th and 9th percentile). Significant differences between lowland and montane (Student's $t$ test, $P<0.01$ ) indicated by a solid circle above panel. (b) Relationship between AGB and elevation (neotropics: red, Asia: blue). Open symbols show sites where no information on slope is available. (c) Relationship between angle of slope and AGB. In (b) and (c), symbols show AGB per land surface area (linear relationship for the neotropics is shown with a dotted line), tops of bars show AGB per planimetric area.
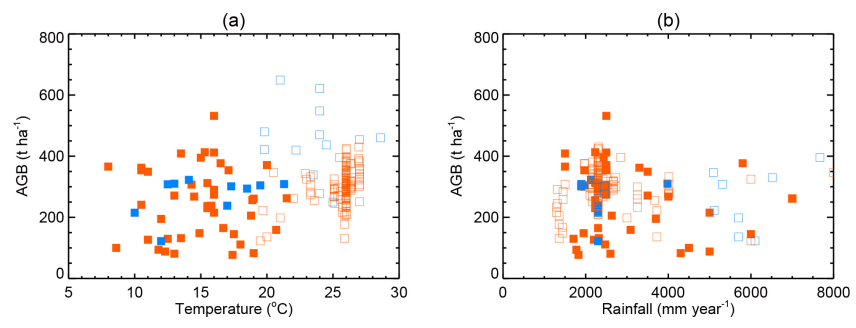

Figure 3. Relationship between AGB (land surface area) and annual mean (a) temperature, (b) rainfall (neotropics: red, Asia: blue). Solid points are for TMFs (elevation $\geq 1000 \mathrm{~m}$ ), open points are for lowland forests.

reported in the studies synthesized here. Future studies of AGB in TMFs need to observe and report a larger suite of environmental parameters.

\subsection{Effects of slope on AGB estimates from forest plots}

Most forest inventory plots are established over a fixed land surface area (Malhi et al., 2006). For example, the RAINFOR protocol uses land surface area as the metric for plot establishment (Phillips et al., 2009). AGB from forest plots is typically reported as the biomass stored per unit area of land surface, whereas the geographical area of forests and remotely sensed forest data are reported on a planimetric basis. Alternatively, AGB can be reported as the biomass stored per planimetric (horizontal) area (Proctor et al., 1983; de Castilho et al., 2006; Alves et al., 2010; Moser et al., 2011; Mascaro et al., 2011). In regions with gentle slopes there is little difference between land surface area and planimetric area so the distinction is often assumed to be unimpor-
Table 2. Correlation $\left(r^{2}\right)$ of above-ground biomass (AGB) with topographical and meteorological variables. Correlations are shown separately for all sites (bold), neotropics (italics) and Asia (normal text) for the subset of sites where data on elevation, slope, temperature and rainfall are all available. Asterisks indicate significant $(P<0.01)$ correlations.

\begin{tabular}{lllllll}
\hline & $\begin{array}{c}\text { AGB } \\
\text { TMF }^{\mathrm{a}}\end{array}$ & $\begin{array}{l}\text { TMF }+ \\
\text { lowland }^{\mathrm{b}}\end{array}$ & $E$ & $S$ & $T$ & $R$ \\
\hline AGB & 1 & 1 & - & - & - & - \\
Elevation $(E)$ & $\mathbf{0 . 0 7}$ & $\mathbf{0 . 0 1}$ & 1 & - & - & - \\
& - & 0.23 & & & & \\
& & 0.42 & & & & \\
Slope $(S)$ & $\mathbf{0 . 1}$ & $\mathbf{0 . 1}{ }^{*}$ & 0.02 & 1 & - & - \\
& - & $0.27^{*}$ & & & & \\
& & 0.14 & & & & \\
Annual mean & $\mathbf{0 . 1 4}$ & $\mathbf{0 . 0 3}$ & 0.96 & 0.01 & 1 & - \\
temperature $(T)$ & - & 0.23 & & & & \\
& & 0.39 & & & & \\
Annual mean & $\mathbf{0 . 2 0}$ & $\mathbf{0 . 0 0 1}$ & 0.02 & 0.06 & 0.05 & 1 \\
rainfall $(R)$ & & 0.08 & & & & \\
& & 0.30 & & & & \\
\hline
\end{tabular}

a TMF only. All sites: $n=24$.

b TMF+ lowland. All sites: $n=111$, neotropics: $n=89$; Asia: $n=19$.

tant. However, on steep slopes the land surface area can be substantially greater than the planimetric surface area, with the ratio being a factor 1.41 on a $45^{\circ}$ slope. This means that biomass storage on a planimetric area basis can be substantially greater than on a land surface area basis.

Across our TMF data set, at sites where slope angle is reported $(n=47)$, the angle varied from 0 to $40^{\circ}$ with a mean slope angle of $17^{\circ}$. This results in AGB storage in our TMF 
plots being, on average, $7 \%$ greater when calculated per unit of planimetric area compared to when calculated per unit of the Earth's surface (Fig. 2b). On the steepest slopes in our data set, AGB on a planimetric surface is $31 \%$ greater than that calculated on a land surface area. In contrast, in the lowland forests plots there is a mean slope of $10^{\circ}(n=90)$ resulting in AGB being on average only $3 \%$ greater when calculated per planimetric surface area as compared to land surface area.

In lowland neotropical forests, previous work has found that slope angle has little (de Castilho et al., 2006) or positive (Mascaro et al., 2011) impact on planimetric AGB. In our analysis (TMF and lowland forests, $n=111$ ), we find a negative correlation between AGB and slope angle as reported in Sect. 3.1. The correlation between AGB (surface area) and slope angle $(r=-0.32)$ is weaker when AGB is calculated on a planimetric basis $(r=-0.25)$. Our analysis extends previous studies (e.g. de Castilho et al., 2006; Mascaro et al., 2011) by including forests plots on slopes with steeper angles and suggests that whilst AGB (planimetric) declines moderately with increasing slope angle, forests on steep slopes (up to angles of $40^{\circ}$ ) still store substantial amounts of biomass.

\subsection{Impacts of slope on regional AGB storage}

The regional and global area of forests is reported as the planimetric surface area. Regional biomass stocks are typically calculated by multiplying the biomass storage per unit area by the planimetric area of the forested region (Gibbs et al., 2007; Baccini et al., 2012). If these calculations use biomass storage per unit land surface area, there is the potential to underestimate regional biomass stocks in forests with steep slopes. In this case, planimetric area should be converted to land surface area before calculating regional biomass storage.

To explore whether slope has implications for the regional biomass stocks of TMFs we combined a high-resolution (7.5 arc sec; $\sim 200 \mathrm{~m}$ pixel at the equator) digital elevation model (DEM) with a global data set of moist tropical forest cover (Hansen et al., 2008). With these data sets, TMF cover 0.88 million $\mathrm{km}^{2}$ (planimetric area) accounting for $8.3 \%$ of total tropical forest area (Fig. 4a). Figure 1 shows the global distribution of tropical forests as a function of elevation. TMFs are distributed across the tropics (47\% the neotropics, $40 \%$ Asia, $13 \%$ Africa) and concentrated in Papua New Guinea, Indonesia, Yunnan Province (China) and throughout the Andes in Central and South America.

For each pixel of the DEM we calculated the angle of slope. We note that variability in elevation at finer spatial scales than the resolution of the DEM mean that our calculated land surface to planimetric area ratios will be a lower limit. The frequency of different slope angles is shown in Fig. 4b. In the lowland tropical forests the average slope angle is $11^{\circ}$ with $50 \%$ of forests having slope angles of less than $9^{\circ}$. Steeper slopes are more frequent in TMFs with the mean slope being $32^{\circ}$ and $75 \%$ of forests having slope angles of greater than $27^{\circ}$. The prevalence of steeper slopes at higher elevations has been shown previously for forests in the Andes, where a transition between flat terrain and steep slopes occurs at around $900 \mathrm{~m}$ elevation (Asner et al., 2014).We note that the global mean slope angle for TMFs $\left(32^{\circ}\right)$ calculated from our remote sensing analysis is greater than the mean slope angle in TMFs from our forest inventory data set $\left(17^{\circ}\right)$, confirming biases in the site selection of forest plots to gentle slopes (Malhi et al., 2006).

We used information on slope angle to calculate the ratio of land surface area to planimetric area across the forested area of the tropics. Figure 5 displays the spatial pattern of this ratio, which reaches a factor of 2 across TMFs of the Andes, Indonesia and Papua New Guinea. These estimates are likely to be a lower limit due to variability in elevation at scales below the resolution of the DEM. Most lowland tropical forests have ratios less than 1.05 although some lowland forest regions of the Amazon Basin (e.g. Guiana Shield) have ratios up to 1.25. Figure $4 \mathrm{c}$ compares the global distribution of this ratio in lowland forests and TMFs. More than $60 \%$ of lowland forests have a surface area to planimetric area of less than 1.05. In TMFs, ratios of up to 2 are common with the most frequent ratio being around 1.4. We find the global land surface area of TMFs is 1.22 million $\mathrm{km}^{2}, 40 \%$ greater than the planimetric area reported above. In our TMF forest inventory plot data set the average ratio of land surface area to planimetric area is only $7 \%$ because the data set is biased to forests with gentle slopes with a paucity of data on steep slopes. In lowland forests, the less steep terrain that is typical here results in only a $7 \%$ difference between land surface area $\left(10.4\right.$ million $\left.\mathrm{km}^{2}\right)$ and planimetric area $\left(9.7\right.$ million $\left.\mathrm{km}^{2}\right)$ of lowland forests.

\section{Conclusions}

We synthesized data of above-ground biomass (AGB) in tropical montane forests (TMFs) (elevation $>1000 \mathrm{~m}$ ) from forest plot inventory studies located in undisturbed forest. We found that mean biomass storage in TMFs was $271 \mathrm{t}$ per hectare of land surface $(n=94)$, significantly less than in lowland tropical forests. The AGB stored by TMFs exhibited substantial variability, with the variability not significantly different from that observed in lowland tropical forests. Widely measured topographical (elevation and slope angle) and climatological (annual mean temperature, annual mean rainfall) parameters only explain a modest fraction of the variability in AGB in TMFs $\left(r^{2}\right.$ typically $\left.<0.2\right)$. Other environmental parameters are therefore likely to be more important in determining AGB and future studies should endeavour to measure a wider suite of environmental parameters.

Our analysis, based on forest plot measurements, is consistent with airborne imaging spectroscopy and lidar studies 
(a)

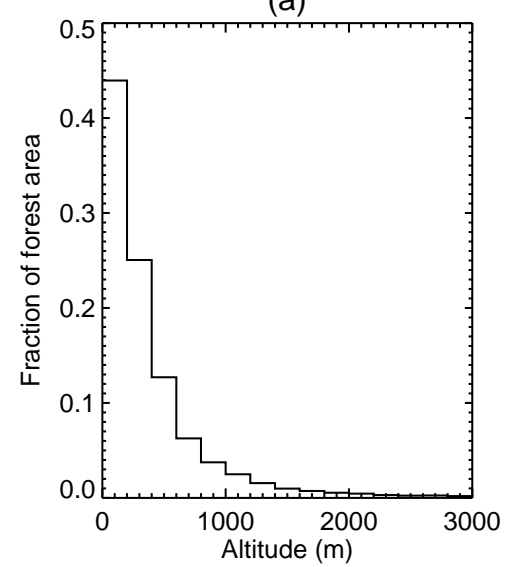

(b)

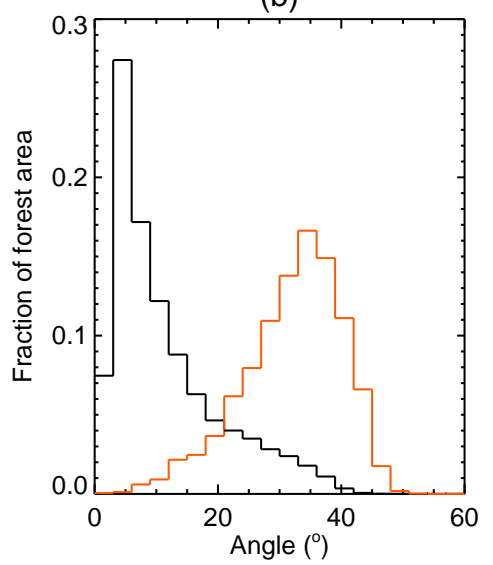

(c)

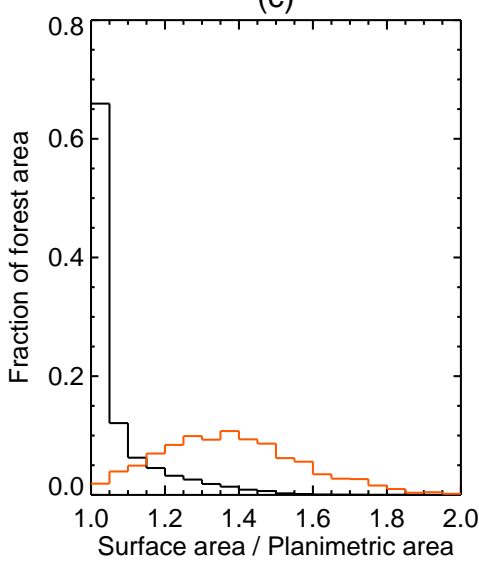

Figure 4. Distribution of the planimetric area of global tropical forests as a function of (a) elevation, (b) slope angle. (c) Ratio of surface area to planimetric area (lowland (elevation $<1000 \mathrm{~m}$ ) tropical forest: black; tropical montane forest (elevation $\geq 1000 \mathrm{~m}$ ): red).

(a)

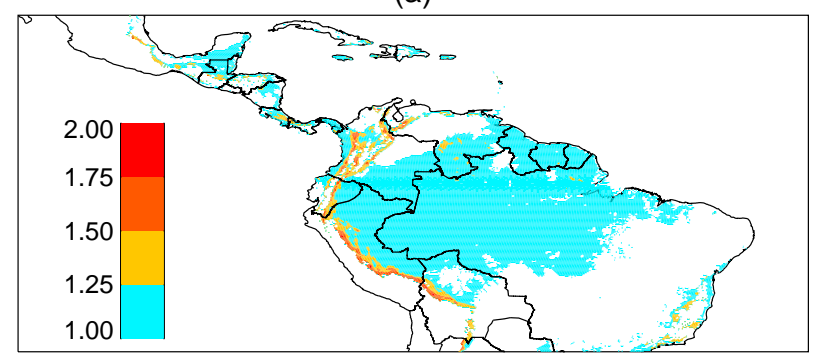

(b)

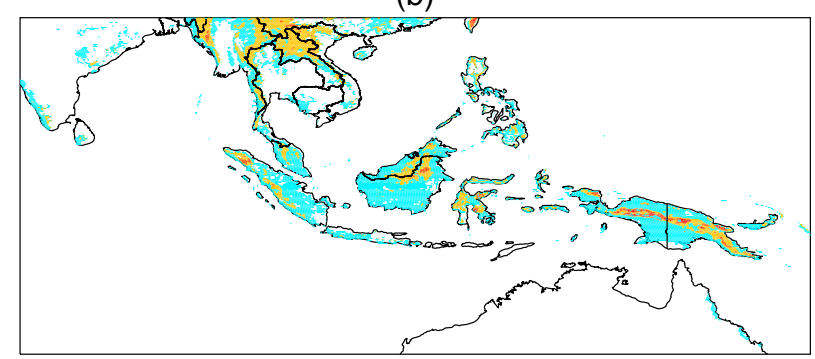

Figure 5. Impact of slope on the area of tropical forests. Colour shows the ratio of land surface area to planimetric area in (a) neotropics, (b) Asia.

quantifying changes in forest structure at a spatial scale of 25 ha along a $3000 \mathrm{~m}$ elevation gradient in the Peruvian Andes (Asner et al., 2014). Such airborne observations promise to be a powerful method to further our understanding of forest structure and AGB along elevation gradients (e.g. Asner et al., 2012, 2014), with errors from this technique of similar magnitude to those from forest plot studies (Mascaro et al., 2011).
We have demonstrated that AGB storage in tropical forests declines moderately with both increasing elevation and slope angle. Despite this, our analysis confirms that TMFs store considerable biomass both at high elevations (up to $3600 \mathrm{~m}$ ) and on steep slopes (slope angles up to $40^{\circ}$ ). On such steep slopes the land surface area is substantially greater than the planimetric area, meaning that estimation of regional biomass storage in montane forests needs to account for slope.

We used remotely sensed data sets of forest cover and elevation to show that $75 \%$ of the planimetric global area of TMFs are on steep slopes (slope angles greater than $27^{\circ}$ ). We used the remotely sensed data sets to demonstrate that this prevalence of steep slopes results in the global land surface area of TMFs (1.22 million $\left.\mathrm{km}^{2}\right)$ being $40 \%$ greater than the planimetric (horizontal) area that is the usual basis for reporting global land surface areas and remotely sensed data.

Our analysis is restricted to AGB as few comparable data are available for below-ground biomass in TMFs. Previous work has documented the importance of below-ground carbon storage within TMF soils (Raich et al., 2006; Leuschner et al., 2007, 2013) which in some cases may exceed AGB stores (Frangi and Lugo, 1985) and will further increase the importance of these ecosystems as a global carbon store. Deforestation and degradation of TMFs are ongoing ( $\mathrm{Ar}-$ menteras et al., 2003; Bruijnzeel et al., 2010; Cayuela et al., 2006). This, combined with the negative implications of future climate change on ecosystem functioning in TMFs (Foster, 2001), highlights the urgent need for conservation attention. Whilst the majority of focus for the role of carbon finance in forest conservation has been on lowland forests, our analysis highlights the significance of TMFs as a global carbon store. 
Acknowledgements. D. V. Spracklen acknowledges a Natural Environment Research Council grant (NE/G015015/1). We acknowledge the US Geological Survey for provision of the GMTED2010 data set. We thank Gregory Asner and Jürgen Homeir for their comments.

Edited by: E. Veldkamp

\section{References}

Acosta-Mireles, M., Vargas-Hernández J., Veláazquez-Martinez A., and Etchevers-Barra, J. D.: Above-ground biomass estimation by means of allometric relationships in six hardwood species in Oaxaca, Mexico, Agrociencia, 36, 725-736, 2002.

Álvarez-Arteaga, G., García Calderón, N. E., Krasilnikov, P., and García-Oliva, F.: Carbon storage in montane cloud forests in Sierra Norte of Oaxaca, Mexico, Agrociencia, 47, 171-180, 2013

Alves, L. F., Vieira, S. A., Scaranello, M. A., Camargo, P. B., Santos, F. A. M., Joly, C. A., and Martinelli, L. A.: Forest structure and live above ground biomass variation along an elevational gradient of topical Atlantic moist forest (Brazil), Forest Ecol. Manag., 260, 679-691, 2010.

Armenteras, D., Gast, F., and Villareal, H.: Andean forest fragmentation and the representativeness of protected natural areas in the eastern Andes, Colombia, Biol. Conserv., 113, 245-256, 2003.

Asner, G. P., Powell, G. V. N., Mascaro, J., Knapp, D. E., Clark, J. K., Jacobson, J., Kennedy-Bowdoin, T., Balaji, A., Paez-Acosta, G., Victoria, E., Secada, L., Valqui, M., and Hughes, R. F.: High-resolution forest carbon stocks and emissions in the Amazon, P. Natl. Acad. Sci. USA, 107, 1673816742, 2010.

Asner, G., Clark, J., Mascaro, J., Vaudry, R., Chadwick, K. D., Vieilledent, G., Rasamoelina, M., Balaji, A., Kennedy-Bowdoin, T., Maatoug, L., Colgan, M., and Knapp, D.: Human and environmental controls over aboveground carbon storage in Madagascar, Carbon Balance Manag., 7, 2, doi:10.1186/1750-0680-7-2, 2012.

Asner, G. P., Anderson, C. B., Martin, R. E., Knapp, D. E., Tupayachi, R., Sinca, F., and Malhi, Y.: Landscape-scale changes in forest structure and functional traits along an Andes-to-Amazon elevation gradient, Biogeosciences, 11, 843-856, doi:10.5194/bg11-843-2014, 2014.

Baccini, A., Goetz, S. J., Walker, W. S., Laporte, N. T., Sun, M., Sulla-Menashe, D., Hackler, J., Beck, P. S. A., Dubayah, R., Friedl, M. A., Samanta, S., and Houghton, R. A.: Estimated carbon dioxide emissions from tropical deforestation improved by carbon-density maps, Nat. Clim. Change, 2, 182-185, 2012.

Baker, T. R., Phillips, O. L., Malhi, Y., Almeida, S., Arroyo, L., Di Fiore, A., Erwin, T., Killeen, T. J., Laurance, S. G., Laurance, W. F., Lewis, S. L., Lloyd, J., Monteagudo, A., Neill, D. A., Patino, S., Pitman, N. C. A., Silva, J. N. M., and Martinez, R. V.: Variation in wood density determines spatial patterns in Amazonian forest biomes, Glob. Change Biol., 10, 545-562, 2004.

Benner, J., Vitousek, P. M., and Ostertag, R.: Nutrient cycling and nutrient limitation in tropical montane cloud forest. in: Tropical montane cloud forests: Science for Conservation and Management, edited by: Bruijnzeel, L. A., Scatena, F. N., and Hamilton, L. S., Cambridge University Press, Cambridge, 90-100, 2010.
Brown, S., Gillespie, A. J. R., Lugo, A. E.: Biomass estimation methods for tropical forests with applications to forest inventory data, 1989.

Bruijnzeel, L. A. and Veneklaas, E. J.: Conditions and tropical montane forest productivity: the fog has not lifted yet, Ecology, 79, 3-9, 1998.

Bruijnzeel, L. A., Kappelle, M., Mulligan, M., and Scatena, F. N.: Tropical montane cloud forests: state of the knowledge and sustainability perspectives in a changing world, in: Tropical montane cloud forests: Science for Conservation and Management, edited by: Bruijnzeel, L. A., Scatena, F. N., and Hamilton, L. S., Cambridge University Press, 691-740, 2010.

Castel, T., Beaudoin, A., Stach, N., Stussi, N., Le Toan, T., and Durand, P.: Sensitivity of space-borne SAR data to forest parameters over sloping terrain, theory and experiment, Int. J. Remote Sens., 22, 2351-2376, 2001.

Cayuela, L., Benayas, J. M. R., and Echeverria, C.: Clearance and fragmentation of tropical montane forests in the Highlands of Chiapas, Mexico (1975-2000), Forest Ecol. Manag., 226, 208218, 2006.

Chave, J., Andalo, C., Brown, S., Cairns, M. A., Chambers, J. Q., Eamus, D., Folster, H., Fromard, F., Higuchi, N., Kira, T., Lescure, J. P., Nelson, B. W., Ogawa, H., Puig, H., Riera, B., and Yamakura, T.: Tree Allometry and Improved Estimation of Carbon Stocks and Balance in Tropical Forests, Oecologia, 145, 87-89, 2005.

Chen, D. X., Li, Y. D., Liu, H. P., Xu, H., Xiao, W. F., Luo, T. S., Zhou, Z., and Lin, M. X.: Biomass and carbon dynamics of a tropical mountain rain forest in China, Sci. China Life Sci., 53 , 798-810, 2010.

Clark, D. A., Brown, S., Kicklighter, D. W., Chambers, J. Q., Thomlinson, J. R., Ni, J., and Holland, E. A.: Net primary production in tropical forests: an evaluation and synthesis of existing field data, Ecol. Appl., 11, 371-384, 2001.

Culmsee, H., Leuschner, C., Moser, G., and Pitopang, R.: Forest aboveground biomass along an elevational transect in Sulawesi, Indonesia, and the role of Fagaceae in tropical montane rain forests, J. Biogeogr., 37, 960-974, 2010.

Cunha, G. D., Gama-Rodrigues, A. C., Gama-Rodrigues, E. F., and Velloso, A. C. X.: Biomass, carbon and nutrient pools in montane Atlantic forests in the North of Rio de Janeiro State, Brazil, Rev. Bras. Cienc. Solo, 33, 1175-1185, 2009.

de Castilho, C. V., Magnusson, W. E., de Araujo, R. N. O., Luizao, R. C. C., Lima, A. P., and Higuchi, N.: Variation in aboveground tree live biomass in a central Amazonian Forest: effects of soil and topography, Forest Ecol. Manag., 234, 85-96, 2006.

Delaney, M., Brown, S., Lugo, A. E., Torres-Lezama, A., and Quintero, N. B.: The distribution of organic carbon in major components of forests located in five life zones of Venezuela, J. Trop. Ecol., 13, 697-708, 1997.

Dislich, C. and Huth, A.: Modelling the impact of shallow landslides on forest structure in tropical montane forests, Ecol. Monitor., 239, 40-53, 2012.

Dossa, G. G. O., Paudel, E., Fujinuma, J., Yu, H., Chutipong, W., Zhang, Y., Paz, S., and Harrison, R. D.: Factors determining forest diversity and biomass on a tropical volcano, Mt. Rinjani, Lombok, Indonesia, PLoS ONE, 8, e67720, doi:10.1371/journal.pone.0067720, 2013. 
Edwards, P. J. and Grubb, P. J.: Studies of mineral cycling in a montane rain forest in New Guinea, I. The distribution of organic matter in the vegetation and soil, J. Ecol., 65, 943-969, 1977.

Feshe, J., Hofstede, R., Aguirre, A., Paladines, C., Kooijman, A., and Sevink, J.: High altitude tropical secondary forests: a competitive carbon sink?, Forest Ecol. Manag., 163, 9-25, 2002.

Fisher, J. B., Malhi, Y., Torres, I. C., Metcalfe, D. B., van de Weg, M. J., Meir, P., Silva-Especjo, and Huasco, W. H.: Nutrient limitation in rainforests and cloud forests along a $3000 \mathrm{~m}$ elevation gradient in the Peruvian Andes, Oecologia, 172, 889-902, 2013.

Foster, P.: The potential negative impacts of global climate change on tropical montane cloud forests, Earth-Sci. Rev., 55, 73-106, 2001.

Frangi, J. L. and Lugo, A. E.: Ecosystem dynamics of a subtropical floodplain forest, Ecol. Monogr., 55, 351-369, 1985.

Gentry, A. H.: Tropical forest biodiversity: distribution patterns and their conservation significance, Oikos, 63, 19-28, 1992.

Gibbon, A., Silman, M. R., Malhi, Y., Fisher, J. B., Meir, P., Zimmermann, M., Dargie, G. C., Farfan, W. R., and Garcia, K. C.: Ecosystem carbon storage across the grassland-forest transition in the high Andes of Manu National Park, Ecosystems, 13, 10971111, 2010.

Gibbs, H. K., Brown, S., Niles, J. O., and Foley, J. A.: Monitoring and estimating tropical forest carbon stocks: making REDD a reality, Environ. Res. Lett., 2, 045023, doi:10.1088/17489326/2/4/045023, 2007.

Girardin, C. A. J., Malhi, Y., Aragão, L. E. O. C., Mamani, M., Huaraca Huasco, W., Durand, L., Feeleys, K. J., Rapp, J., SilvaEspejo, J. E., Silman, M., Salinas, N., and Whittaker, R. J.: Net primary productivity allocation and cycling of carbon along a tropical forest elevational transect in the Peruvian Andes, Glob. Change Biol., 16, 3176-3192, doi:10.1111/j.13652486.2010.02235.x, 2010.

Girardin, C. A. J., Aragão, L. E. O. C., Malhi, Y., Huaraca Huasco, W., Metcalfe, D., and Silvae, J. E.: Fine root dynamics along an elevational gradient in tropical Amazonian and Andean forests, Global Biogeochem. Change, 27, 252-264, 2013.

Girardin, C. A. J., Farfan-Rios, W., Garcia, K., Feeley, K. J., Jørgensen, P. M., Murakami, A. A., Pérez, L. C., Seidel, R., Paniagua, N., Claros, A. F. F., Maldonado, C., Silman, M., Salinas, N., Reynel, C., Neill, D. A., Serrano, M., Caballero, C. J., La Torre-Cuadros, M. D. L. A., Macìa, M. J., Killeen, T. J., and Malhi, Y.: Spatial patterns of above-ground structure, biomass and composition in a network of six Andean elevation transects, Plant Ecol. Divers., 1-11, doi:10.1080/17550874.2013.820806, 2014a.

Girardin, C. A. J., Espejob, J. E. S., Doughty, C. E., Huasco, W. H., Metcalfe, D. B., Durand-Baca, L., Marthews, T. R., Aragao, L. E. O. C., Farfàn-Rios, W., Garcìa-Cabrera, K., Halladay, K., Fisher, J. B., Galiano-Cabrera, D. F., Huaraca-Quispe, L. P., AlzamoraTaype, I., Eguiluz-Mora, L., Salinas-Revilla, N., Silman, M. R., Meir, P., and Malhi, Y.: Productivity and carbon allocation in a tropical montane cloud forest of the Peruvian Andes, Plant Ecol. Divers., 7, 107-123, 2014b.

Grimm, U. and Fassbender, H. W.: Ciclos bioquímicos en un ecosistema forestal de los Andes Occidentales de Venezuela, I. Inventario de las reservas organicas y minerales $(\mathrm{N}, \mathrm{P}, \mathrm{K}, \mathrm{Ca}, \mathrm{Mg}$, Mn, Fe, Al, Na), Turrialba, 31, 27-37, 1981.
Hansen, M., DeFries, R. S., Townshend, J. R. G., Carroll, M., Dimiceli, C., and Sohlberg, R. A.: Global percent tree cover at a spatial resolution of 500 meters: first results of the MODIS Vegetation Continuous Fields Algorithm, Earth Interact., 7, 1-15, 2003.

Hansen, M. C., Stehman, S. V., Potapov, P. V., Loveland, T. R., Townshend, J. R. G., DeFries, R. S., Pittman, K. W., Arunarwati, B., Stolle, F., Steininger, M. K., Carroll, M., and DiMiceli, C.: Humid tropical forest clearing from 2000 to 2005 quantified using multi-temporal and multi-resolution remotely sensed data, P. Natl. Acad. Sci. USA, 105, 9439-9444, 2008.

Hertel, D., Moser, G., Culmsee, H., Erasmi, S., Horna, V., Schuldt, B., and Leuschner, Ch.: Below- and above-ground biomass and net primary production in a paleotropical natural forest (Sulawesi, Indonesia) as compared to neotropical forests, Forest Ecol. Manag., 258, 1904-1912, 2009.

Hofstede, R. and Aguirre, N.: Biomasa y dinámica del carbono en relación con las actividades forestales en la Sierra del Ecuador, in: El Páramo Como Espacio de Mitigación de Carbono Atmosferico, Serie Páramo 1, edited by: Medina, G. and Mena, P., Ediciones Abya Yala, Quito, 29-51, 1999.

Homeier, J., Hertel, D., Camenzind, T., Cumbicus, N. L., Maraun, M., Martinson, G. O., Poma, L. N., Rillig, M. C., Sandmann, D., Scheu, S., Veldkamp, E., Wilcke, W., Wullaert, H., and Leuschner, C.: Tropical Andean forests are highly susceptible to nutrient inputs - Rapid effects of experimental $\mathrm{N}$ and $\mathrm{P}$ addition to an Ecuadorian Montane Forest, PLoS ONE, 7, e47128, doi:10.1371/journal.pone.0047128, 2013.

Kitayama, K. and Aiba, S. I.: Ecosystem structure and productivity of tropical rain forests along altitudinal gradients with contrasting soil phosphorus pools on Mount Kinabalu, Borneo, J. Ecol., 90, 37-51, 2002.

Leuschner, C., Moser, G., Bertsch, C., Roderstein, M., and Hertel, D.: Large altitudinal increase in tree root/shoot ratio in tropical mountain forests of Ecuador, Basic Appl. Ecol., 8, 219-230, 2007.

Leuschner, C., Zach, A., Moser, G., Homeier, J., Graefe, S., Hertel, D., Wittich, B., Soethe, N., Iost, S., Röpderstein, M., Horna, V., and Wolf, K.: The carbon balance of tropical mountain forests along an altitudinal transect, in: Ecological Studies Vol. 221, edited by: Bendix, J., Springer Verlag, Berlin, Heidelberg, New York, 10, 117-139, 2013.

Lewis, S. L., Sonke, B., Sunderland, T., Begne, S. K., LopezGonzalez, G., van der Heijden, G. M. F., Phillips, O. L., AffumBaffoe, K., Baker, T. R., Banin, L., Bastin, J.-F., Beeckman, H., Boeckx, P., Bogaert, J., De Canniere, C., Chezeaux, E., Clark, C. J., Collins, M., Djagbletey, G., Djuikouo, M. N. K., Droissart, V., Doucet, J.-L., Ewango, C. E. N., Fauset, S., Feldpausch, T. R., Foli, E. G., Gillet, J.-F., Hamilton, A. C., Harris, D. J., Hart, T. B., de Haulleville, T., Hladik, A., Hufkens, K., Huygens, D., Jeanmart, P., Jeffery, K. J., Kearsley, E., Leal, M. E., Lloyd, J., Lovett, J. C., Makana, J.-R., Malhi, Y., Marshall, A. R., Ojo, L., Peh, K. S.-H., Pickavance, G., Poulsen, J. R., Reitsma, J. M., Sheil, D., Simo, M., Steppe, K., Taedoumg, H. E., Talbot, J., Taplin, J. R. D., Taylor, D., Thomas, S. C., Toirambe, B., Verbeeck, H., Vleminckx, J., White, L. J. T., Willcock, S., Woell, H., and Zemagho, L.: Above-ground biomass and structure of 260 African tropical forests, Phil. T. R. Soc. B, 368, 1625, doi:10.1098/rstb.2012.0295, 2013. 
Lieberman, D., Lieberman, M., Peralta, R., and Hartshorn, G. S.: Tropical forest structure and composition on a large-scale altitudinal gradient in Costa Rica, J. Ecol., 84, 137-152, 1996.

Malhi, Y., Wood, D., Baker, T. R., Wright, J., Phillips, O. L., Cochrane, T., Meir, P., Chave, J., Almeida, S., Arroyo, L., Higuchi, N., Killeen, T. J., Laurance, S., G., Laurance, W. F., Lewis, S. L., Monteagudo, A., Neill, D. A., Vargas, P. N., Pitman, N. C. A., Quesada, C. A., Salomão, R., Silva, J. N. M., Lezama, A. T., Terborgh, J., Martínez, R. V., and Vinceti, B.: The regional variation of aboveground live biomass in old-growth Amazonian forests, Glob. Change Biol., 12, 1107-1138, 2006.

Martínez, M. L., Pérez-Maqueo, O., Vázquez, G., CastilloCampos, G., García-Franco, J., Mehltreter, K., Equihua, M., and Landgrave, R.: Effects of land use change on biodiversity and ecosystem services in tropical montane cloud forests of Mexico, Forest Ecol. Manag., 258, 1856-1863, 2009.

Mascaro, J., Asner, G. P., Muller-Landau, H. C., van Breugel, M., Hall, J., and Dahlin, K.: Controls over aboveground forest carbon density on Barro Colorado Island, Panama, Biogeosciences, 8, 1615-1629, doi:10.5194/bg-8-1615-2011, 2011.

Moser, G., Hertel, D., and Leuschner, C.: Altitudinal change in LAI and stand leaf biomass in tropical montane forests: a transect study in ecuador and a Pan - Tropical meta-analysis, Ecosystems, 10, 924-935, 2007.

Moser, G., Leuschner, C., Hertel, D., Graefe, S., Soethe, N., and Iost, S.: Elevation effects on the carbon budget of tropical mountain forests (S Ecuador): the role of the belowground compartment, Glob. Change Biol., 17, 2211-2226, 2011.

Nadkarni, N. M., Schaefer, D., Matelson, T. J., and Solano, R.: Biomass and nutrient pools of canopy and terrestrial components in a primary and a secondary montane cloud forest, Costa Rica, Forest Ecol. Manag., 198, 223-236, 2004.

Ovington, J. D. and J. S. Olson: Biomass and chemical content of El Verde lower montane rain forest, Chapter H-2, in: A tropical rain forest, edited by: Odum, H. T. and Pigeon, R. F., United States Atomic Energy Commission, National Technical Information Service, Springfield, Virginia, USA, 1970.

Omoro, L. M. A., Starr, M., and Pellikka, P. K. E.: Tree biomass and soil carbon stocks in indigenous forests in comparison to plantations of exotic species in the Taita Hills of Kenya, Silva Fenn., 47, 1-18, 2013.

Paoli, G. D., Curran, L. M., and Slik, J. W. F.: Soil nutrients affect spatial patterns of aboveground biomass and emergent tree density in southwestern Borneo, Oecologia, 155, 287-299, 2008.

Phillips, O., Baker, T., Feldpausch, T., and Brienen, R.: RAINFOR field manual for plot establishment and remeasurement, available at: http://www.rainfor.org/upload/ManualsEnglish/RAINFOR_ field_manual_version_June_2009_ENG.pdf (last access: 15 November 2013), 2009.

Proctor, J., Anderson, J. M., Chai, P., and Vallack, H. W.: Ecological studies in four contrasting lowland rain forests in Gunung Mulu National Park, Sarawak: I. forest environment, structure and floristics, J. Ecol., 71, 237-260, 1983.

Rai, S. N. and Proctor, J.: Ecological studies on four rainforests in Karnataka, India, J. Ecol., 74, 439-454, 1986.

Raich, J. M., Russell, A. E., and Vitousek, P. M.: Primary productivity and ecosystem development along an elevational gradient on Manua Loa, Hawaii, Ecology, 78, 707-721, 1997.
Raich, J. W., Russell, A. E., Kitayama, K., Parton, W. J., and Vitousek, P. M.: Temperature influences carbon accumulation in moist tropical forests, Ecology, 87, 76-87, 2006.

Robert, A.: Simulation of the effect of topography and tree falls on stand dynamics and stand structure of tropical forests, Ecol. Monitor., 167, 287-303, 2003.

Román-Cuesta, R. M., Salinas, N., Asbjornsen, H., Oliveras, I., Huaman, V.Gutiérrez, Y., Puelles, L., Kala, J., Yabar, D., Rojas, M., Astete, R., Jordán, D. Y., Silman, M., Mosandl, R., Weber, M., Stimm, B., Günter, S., Knoke, T., and Malhi, Y.: Implications of fires on carbon budgets in Andean cloud montane forest: the importance of peat soils and tree resprouting, Forest Ecol. Manag., 261, 1987-1997, 2011.

Saatchi, S. S., Houghton, R. A., dos Santos Alvalá, R. C., Soares, J. V., and Yu, Y.: Distribution of aboveground live biomass in the Amazon basin, Glob. Change Biol., 13, 816-837, 2007.

Saatchi, S. S., Harris, N. L., Brown,, S., Lefsky, M., Mitchard, E. T. A., Salas, W., Zutta, B. R., Buermann, W., Lewis, S L., Hagen, S., Petrova, S., White, L., Silman, M., and Morel, A.: Benchmark map of forest carbon stocks in tropical regions across three continents, P. Natl. Acad. Sci. USA, 108, 98999904, doi:10.1073/pnas.1019576108, 2011.

Scatena, F. N., Silver, W., Siccama, T., Johnson, A., and Sanchez, M. J.: Biomass and nutrient control of the Bisley Experimental Watersheds, Luquillo Experimental Forest, Puerto Rico, before and after Hurricane Hugo, 1989, Biotropica, 25, 15-27, 1993.

Sierra, C. A., del Valle, J. I., Orrego, S. A., Moreno, F. H., Harmon, M. E., Zapata, M., Colorado, G. J., Herrera, M. A., Lara, W., Restrepo, D. E., Berrouet, L. M., Loaiza, L. M., and Benjumea, J. F.: Total carbon stocks in a tropical forest landscape of the Porce region, Colombia, Forest Ecol. Manag., 243, 299-309, 2007.

Simard, M., Pinto, N., Fisher, J. B., and Baccini, A.: Mapping forest canopy height globally with spaceborne lidar, J. Geophys. Res., 116, G04021, doi:10.1029/2011JG001708, 2011.

Slik, J. W. F., Aiba, S. I., Brearley, F. Q., Cannon, C. H., Forshed, O., Kitayama, K., Nagamasu, H., Nilus, R., Payne, J., Paoli, G., Poulsen, A. D., Raes, N., Sheil, D., Sidiyasa, K., Suzuki, E., and van Valkenburg, J. L. C. H.: Environmental correlates of tree biomass, basal area, wood specific gravity and stem density gradients in Borneo's tropical forests, Global Ecol. Biogeogr., 19, 50-60, 2010.

Slik, J. W. F., Paoli, G., McGuire, K., Amara, I., Barroso, J., Bastian, M., Blanc, L., Bongers, F., Boundja, P., Clark, C., Collins, M., Dauby, G., Ding, Y., Doucet, J.-L., Eler, E., Ferreira, L., Forshed, O., Fredriksson, G., Gillet, J.-F., Harris, D., Leal, M., Laumonier, Y., Malhi, Y., Mansor, A., Martin, E., Miyamoto, K., Nagamasu, A. A.-M., H., Nilus, R., Nurtjahya, E., Oliveira, A., Onrizal, O., Parada-Gutierrez, A., Permana, A., Poorter, L., Poulsen, J., Ramirez-Angulo, H., Reitsma, J., Rovero, F., Rozak, A., Sheil, D., Silva-Espejo, J., Silveira, M., Spironelo, W., ter Steege, H., Stevart, T., Navarro-Aguilar, G. E., Sunderland, T., Suzuki, E., Tang, J., Theilade, I., van der Heijden, G., van Valkenburg, J., Van Do, T., Vilanova, E., Vos, V., Wich, S., Wöll, H., Yoneda, T., Zang, R., Zhang, M.-G., and Zweifel, N.: Large trees drive forest aboveground biomass variation in moist low- 
land forests across the tropics, Global Ecol. Biogeogr., 22, 12611271, doi:10.1111/geb.12092, 2013.

Spracklen, D. V. and Righelato, R.: Carbon storage and sequestration in tropical montane forests of southern Ecuador, World Land Trust, Halesworth, Suffolk, UK, 2005.

Tanner, E. V. J.: Studies on the biomass and productivity in a series of montane rain forests in Jamaica, J. Ecol., 68, 573-588, 1980.

Unger, M., Homeier, J., and Leuschner, C.: Effects of soil chemistry on tropical forest biomass and productivity at different elevations in the equatorial Andes, Oecologia, 170, 263-274, doi:10.1007/s00442-012-2295-y, 2012.
Unger, M., Homeier, J., and Leuschner, C.: Relationships among leaf area index, below-canopy light availability and tree diversity along a transect from tropical lowland to montane forests in NE Ecuador, Tropical Ecol., 54, 33-45, 2013.

Weaver, P. L. and Murphy, P. G.: Forest structure and productivity in Puerto Rico's Luquillo Mountains, Biotropica, 22, 69-82, 1990.

Yamakura, T., Hagihara, A., Sukardjo, S., and Ogawa, H.: Aboveground biomass of tropical rain forest stands in Indonesian Borneo, Vegetatio, 68, 71-82, 1986. 